\title{
Hot-Electron Tunneling Sensors for High-Resolution X-ray and Gamma-ray Spectroscopy
}

\author{
C. A. Mears \\ S. E. Labov \\ M. Frank \\ H. Netel
}

February 7, 1997

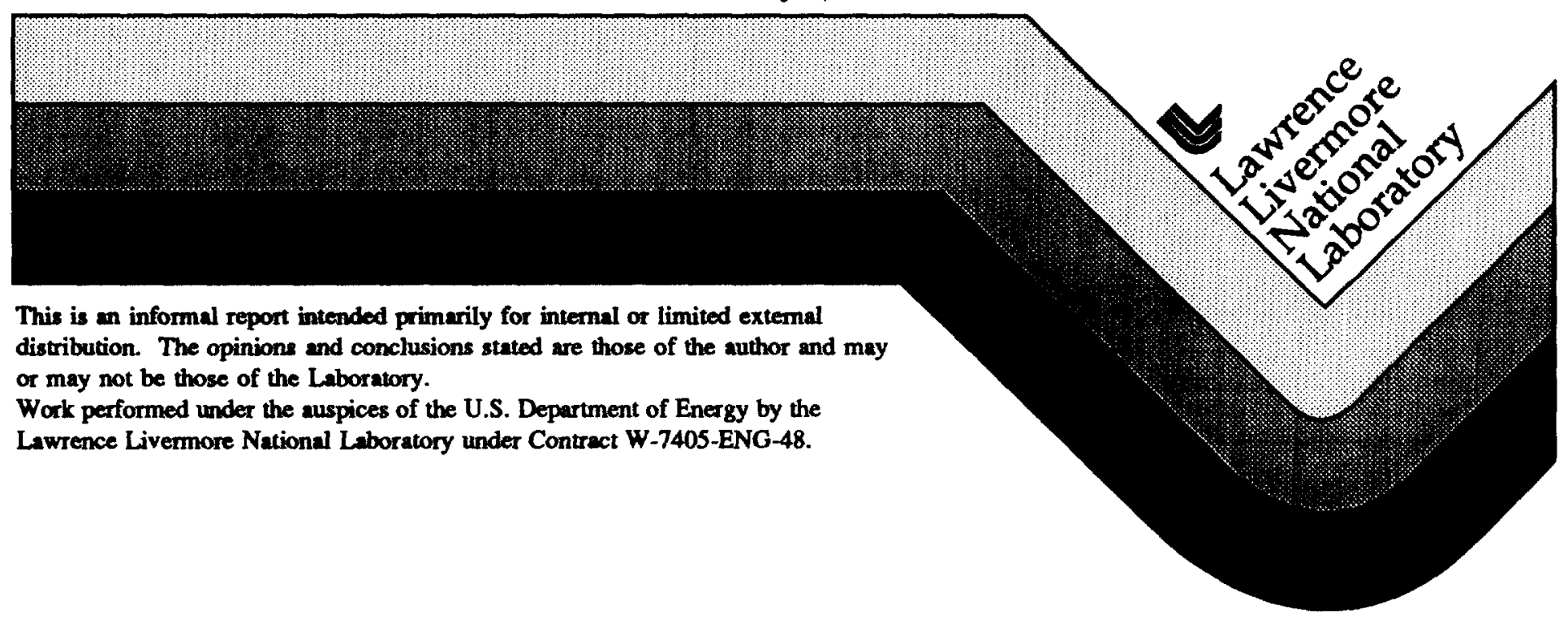




\section{DISCLAMER}

This document was prepared as an acoount of work sponsored by an agency of the United States Government. Neither the United States Government nor the Unlversity of California nor any of their employees, makes any warranty, express or implied, or asoumes any legal liability or responsibility for the accuracy, completeness, or usefulness of any information, apparatus, product, or process disclosed, or represents that its use would not infringe privately owned rights. Reference herein to any speclfic commercial product, process, or service by trade name, trademark, manufacturer, or otherwise, does not necessarily constitute or imply its endorsement, recommendation, or favoring by the United States Covernment or the University of California. The views and opinions of authors expressed herein do not necessarily state or reflect those of the United States Covemment or the University of California, and shall not be used for advertising or product endorsement purposes.

This repart has been reproduced directly from the best available copy.

Available to DOE and DOE contractors from the Office of Scientific and Technical Information P.O. Box 62, Oak Ridge, IN 37831

Prices available from (615) 576-8401, FIS 626-8401

Available to the public from the National Technical Information Service

U.S. Depertment of Commerce 5285 Port Royal Rd. Springfield, VA 22161 


\title{
Hot-Electron Tunneling Sensors for High-Resolution X-ray and Gamma-ray Spectroscopy
}

\author{
Carl A. Mears, Simon E. Labov, Matthias Frank, H. Netel \\ Astrophysics Group, Physics and Space Technology Directorate \\ Lawrence Livermore National Laboratory \\ Livermore, CA 94551
}

\begin{abstract}
Over the past two years we have been studying the use of Hot Electron Tunneling (HET) sensors for use in high-energy-resolution X-ray and gamma-ray spectrometers. These sensors promise several advantages over existing cryogenic sensors, including simultaneous high count rate and high resolution capability, and relative ease of use. Using simple shadow mask lithography techniques, we verified the basic principles of operation of these devices, and discovered new physics in their thermal behavior as a function of applied voltage bias. We also began to develop ways to use this new sensor in practical X-ray and gamma-ray detectors based on superconducting absorbers. This requires the use of quasiparticle trapping to concentrate the signal in the sensing elements.
\end{abstract}




\section{Introduction}

Efficient, broad-band X-ray and gamma-ray spectrometers with high energy resolution are not currently available even though they are highly desirable for a wide range of applications in nuclear physics, particle physics, astrophysics, materials science, chemistry and biology. Common X-ray spectrometers are divided into two types. Those spectrometers with high efficiency, such as scintillation and ionization detectors, have limited resolution. Those spectrometers which use Bragg crystals or gratings have very high energy resolution, but sacrifice efficiency, bandwidth, and ease of use. For gamma rays, the current situation is even worse, since diffraction is not an option. Here, any increase in energy resolution would be extremely useful to the scientific community.

Cryogenic X-ray and gamma-ray spectrometers will combine efficiency, broad bandwidth and high energy resolution. The development of an efficient, broad-band, energy-resolving spectrometer will revolutionize X-ray and gamma-Ray spectrometry. Once ready for use, these detectors will be used in many applications in the Lab, especially in treaty verification work, where improved energy resolution for gamma-rays will aid identification of radionuclides. These detectors will also be used in many laboratory programs involving $X$-ray spectroscopy, in the DOE as a whole, and after commercialization, in the scientific and industrial community at large.

During the last two years, we have undertaken the evaluation of a new type of device, the hot electron tunneling (HET) sensor, that has been proposed for cryogenic spectrometers. This new sensor, allows us to overcome many of the difficulties that have been encountered in the development of high-performance detectors.

\section{Background and Motivation}

\section{A. Cryogenic Detectors}

Many types of cryogenic detectors have been proposed which utilize a variety of absorbing materials and sensing techniques ${ }^{1}$. All these detectors share a common operating principle. The X-ray or gamma-ray photon is absorbed in an ultra-pure sample of an insulator, a semiconductor, or a superconductor, where it deposits its energy. This energy creates a large number of nonequilibrium phononic or electronic excitations. Because of the low temperature and purity of the sample, these excitations live long enough to diffuse into sensing elements where they are detected. The sensors are typically so cold that the energy deposited in them is comparable to the total energy in their thermal fluctuations, which makes it possible to measure the deposited energy with high precision.

There are already several types of cryogenic sensing elements under development, both at LLNL and elsewhere. These include superconducting-insulating-superconducting (SIS) tunnel junctions ${ }^{1-3}$, and microcalorimeters ${ }^{4,5}$. SIS tunnel junctions measure the number 
of Cooper pairs broken by the X-ray interaction by noting an increase in the number of electrons tunneling through a thin insulating layer. Microcalorimeters measure the temperature rise in a small sample of material using a thermal sensor, such as NTD germanium, ion-implanted silicon or superconducting phase-transition sensor.

Our group at LLNL has been developing SIS tunnel junction detectors with much success -- we hold the world record ( $29 \mathrm{eV}$ FWHM at $6 \mathrm{keV}$, and $5 \mathrm{eV}$ at $300 \mathrm{eV}$ ) for energy resolution for this type of detector. The SIS tunnel junction detector has many advantages over microcalorimeters. Tunnel junctions are constructed using standard microfabrication techniques. This makes them easy to fabricate reproducibly (if we can make one, we can make thousands) and easy to arrange into arrays to provide imaging and increased active area (if we can make one, we can make thousands on the same wafer). Also, the thermalization of the absorbed energy is extremely rapid in these devices, making them capable of much higher count rates than microcalorimeters. In certain configurations, SIS tunnel junction detectors can be used as imaging detectors with spatial resolution as good as $5 \mu \mathrm{m}^{6}$.

Despite these advantages, we have found these devices difficult to operate due to bias instabilities, and due to their sensitivity to fluctuations in the magnetic field that must be applied for them to operate. These difficulties originated from the presence of the Josephson effect, caused by the tunneling of paired electrons (Cooper pairs) between the two superconducting electrodes.

Recently, a new sensor has been proposed 7,8 that is free from the Josephson effect, yet retains all the advantages of the SIS sensor. This new sensor is based on a measurement of the temperature of electrons heated by thermalization of non-equilibrium excitations in a small normal metal sensor. This is done by measuring the temperature-dependent tunneling current through a normal-insulating-superconducting (NIS) tunnel junction. This hot-electron tunneling (HET) sensor has the excellent energy resolution common to all cryogenic sensor candidates. Unlike its competition, the HET sensor simultaneously offers high count-rate capability, a broad range of operating temperatures, insensitivity to fluctuations in magnetic field, freedom from erratic behavior due to the Josephson effect, and direct fabrication using standard lithographic techniques. They also easily can be configured into large arrays. In addition to these generic advantages, the HET sensor promises several advantages applicable to our mission to develop high-resolution cryogenic gamma-ray spectrometers for use in arms control and treaty verification applications. Our spectrometers, which are still under development, are based on large, ultrapure superconducting crystals. The HET sensor provides an efficient and rapid way to collect the energy deposited in these large crystals into sensing elements via the use of several levels of quasiparticle trapping. Quasiparticle trapping will be discussed in detail in section III B. The HET sensor also requires no magnetic field for stable operation. An applied magnetic field would degrade the desirable superconducting properties of our superconducting crystals 


\section{Principle of operation}

\section{A. The cryogenic detector}

A schematic diagram of a prototypical cryogenic detector is show in Figure 3.1. These devices operate in the following manner. The quanta to be measured (X-ray or gammaray photon, or energetic particle) is absorbed in the absorber. Here its energy is used to create a cascade of excitations. After a short time ( $<1 \mathrm{~ns})$, most of the energy is contained in a large number of solid state excitations, including phonons and excited electronic states. In order to have an energy resolution better than that exhibited by semiconductor ionization detectors, the number of these solid state excitations must be much greater that the number of electron-hole pairs created in those detectors. This requires that the excitation energy of these excitations be much less than the eV-scale creation energy for an electron-hole pair in a typical semiconductor. 'This is exactly the case both for phonons, and for the quasiparticles formed when the paired electrons in $\mathrm{s}$ superconductor, called Cooper pairs, are broken. Both of these types of excitations have typical energies in the meV range, leading to an increase in the number of excitations by a factor of $\sim 1000$ when compared to a semiconductor-based detector. If the energy resolution of the detector is limited by statistical fluctuations in the number of excitations created by the absorption of the quanta to be measured, this increase in the number of excitations should lead to an improvement in the energy resolution by a factor of approximately $(1000)^{1 / 2}$ or 30 . Such an increase in resolving power in an energy dispersive spectrometer will revolutionize many field of spectroscopy by making possible many previously impossible measurements and experiments.

The low excitation energy of these phonons and quasiparticles makes it necessary to operate the detectors at very low temperature. This is both to ensure that the excitations created have long enough lifetimes so that their number can be accurately measured, and to keep the number of thermally excited excitations low enough so that they do not degrade the accuracy to which the number of newly created excitations can be measured. Typically cryogenic detectors are operated at temperatures below $1.0 \mathrm{~K}$, and often are operated at temperatures as low as $0.1 \mathrm{~K}$.

In our group we are developing cryogenic detectors based on superconducting absorbers. To a reasonable approximation, the important excitation in these absorbers are quasiparticles, or broken Cooper pairs. For many of the discussions below, we ignore the contributions due to excited phonons, though in some cases they to may be important, and will be discussed in those cases.

\section{B. Quasiparticle Trapping}

In order for an X-ray or gamma-ray spectrometer to be useful in the scientific community, its absorber must be large enough to absorb a significant number of the photons emitted by the source under study. On the other hand, the size of the sensor used to detect the excitations is limited by the detrimental effects of sensor parameters that scale with sensor size such as total heat capacity or electrical capacitance, on our ability 


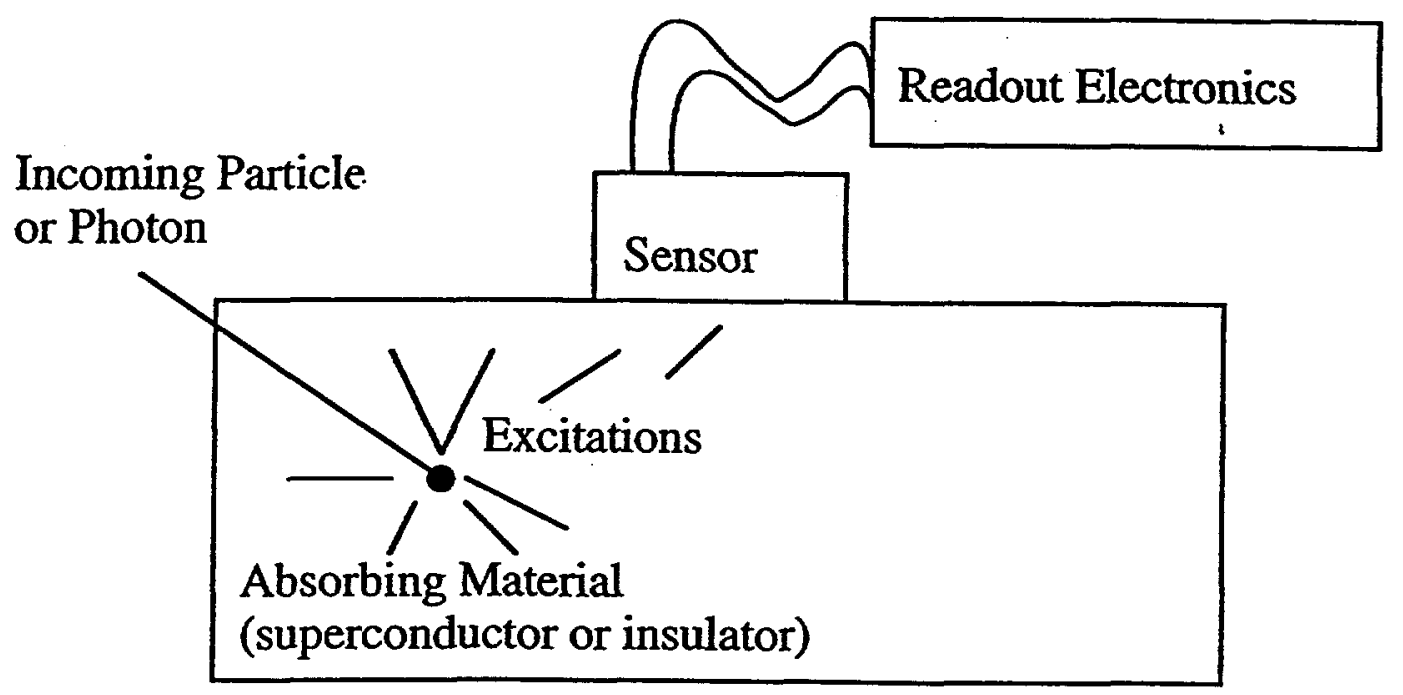

Figure 3.1. Schematic diagram of a cryogenic detector. The incoming quanta to be measured is absorbed in the absorbing material, where it creates millions of low-energy solid-state excitations. These excitations diffuse into the sensor, where their total number and/or total energy is measured. 
to accurately measure the number of excitations. For these reasons, the volume of the sensor may be orders of magnitude less than the volume of the superconducting absorber. Unlike the case of a semiconductor ionization detector, we cannot sweep the excitations to the sensor using an electric field -- the quasiparticles diffuse randomly throughout the absorbing material. Still, the excitations must be concentrated in the sensor region, or the measured signal will be too small and too slow for accurate measurements. In order to achieve this desired concentration, we use a process called quasiparticle trapping?

The energy required to create a quasiparticle in a superconductor, known as the energy gap, varies from one superconducting material to another. By placing different superconducting materials which have different values of the energy gap in close contact, we can use the superconducting version of "bandgap engineering" to concentrate quasiparticles in the material with the lower values of the energy gap. A schematic diagram to help explain this process is shown in Figure 3.2. Quasiparticles are created in the material with the larger energy gap. Quasiparticles diffuse into the material with the lower gap. These quasiparticles can relax to this low gap by emitting a phonon, as shown in the diagram. Once they have relaxed to this lower level they can no longer return to the region of higher gap, and are thus trapped in the lower gap region. If the volume of the lower gap region is less than that of the higher gap region, the quasiparticles are significantly concentrated by this process.

\section{The HET Sensor}

An HET sensor consists of a normal-metal electrode, which serves as a trap for incoming quasiparticles, and a normal-insulating-superconducting (NIS) tunnel junction, which serves to measure the temperature of the electrons in the normal metal. The NIS tunnel junction, which we show schematically in Figure 3.3, operates as follows. When biased at a voltage $V_{b}$, only those electrons whose energy above the Fermi level is greater than $\left(\Delta-\mathrm{eV}_{\mathrm{b}}\right)$ can tunnel, where $\Delta$ is the superconducting gap energy in the superconducting electrode. The number of such electrons is exponentially dependent on the temperature of the hot electrons, making the junction an excellent thermometer for the electron system $^{8}$. When energy in the form of quasiparticles enters the normal metal electrode, it is quickly thermalized via electron-electron and electron-phonon interactions ${ }^{10}, 11$, and the electron temperature is increased. At low temperatures, $\mathrm{T} \leq 0.1 \mathrm{~K}$, the electrons are sufficiently decoupled from the phonon heat bath that they remain warm long enough (several microseconds) for their temperature to be accurately measured by noting the amplitude of the current pulse that the heating of these electrons produces. This temperature change is proportional to the amount of energy arriving from the quasiparticle system in the superconducting absorber.

In order for the HET sensor to be useful, the precision with which it can measure this incoming energy must be high enough. The energy resolution of the HET sensor is limited by thermal fluctuations in the normal metal sensor, and by electronic noise in the tunneling process and in the amplifier used to measure the tunneling current. Taking these noise sources into account, the energy resolution $\Delta E$ is ${ }^{7}$ 


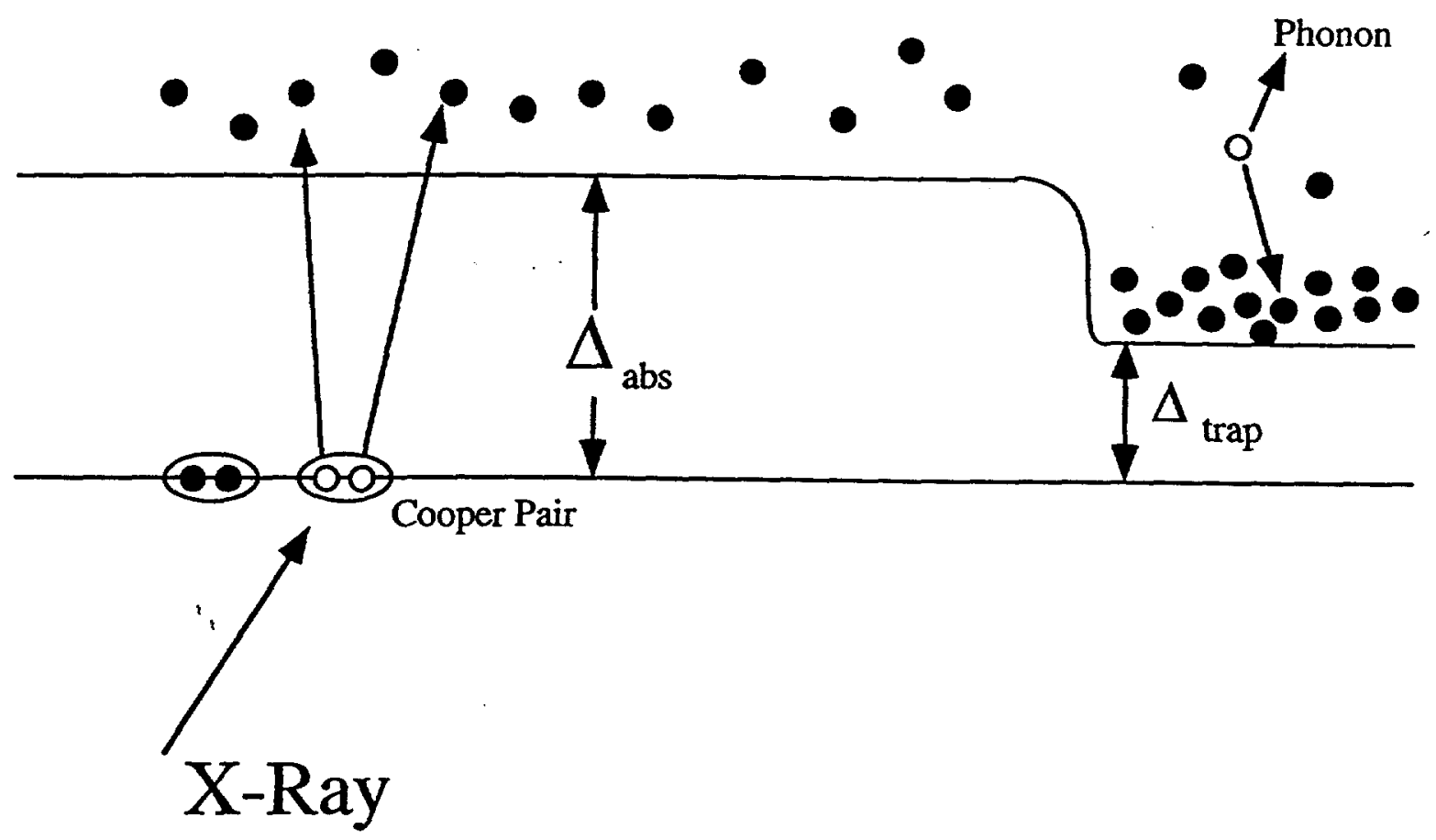

Figure 3.2. The use of quasiparticle trapping to concentrate photon-induced quasiparticles. An X-ray or gamma-ray photon is absorbed in a high-gap superconductor where it results in the production of millions of quasiparticles. These quasiparticles diffuse into the trapping region, made from a lower gap superconductor. Here, they can lose energy by emitting a phonon. After they have relaxed to a state near this lower energy gap, it is energetically impossible for them to return to the high gap region, and thus they are trapped and concentrated in this region. 


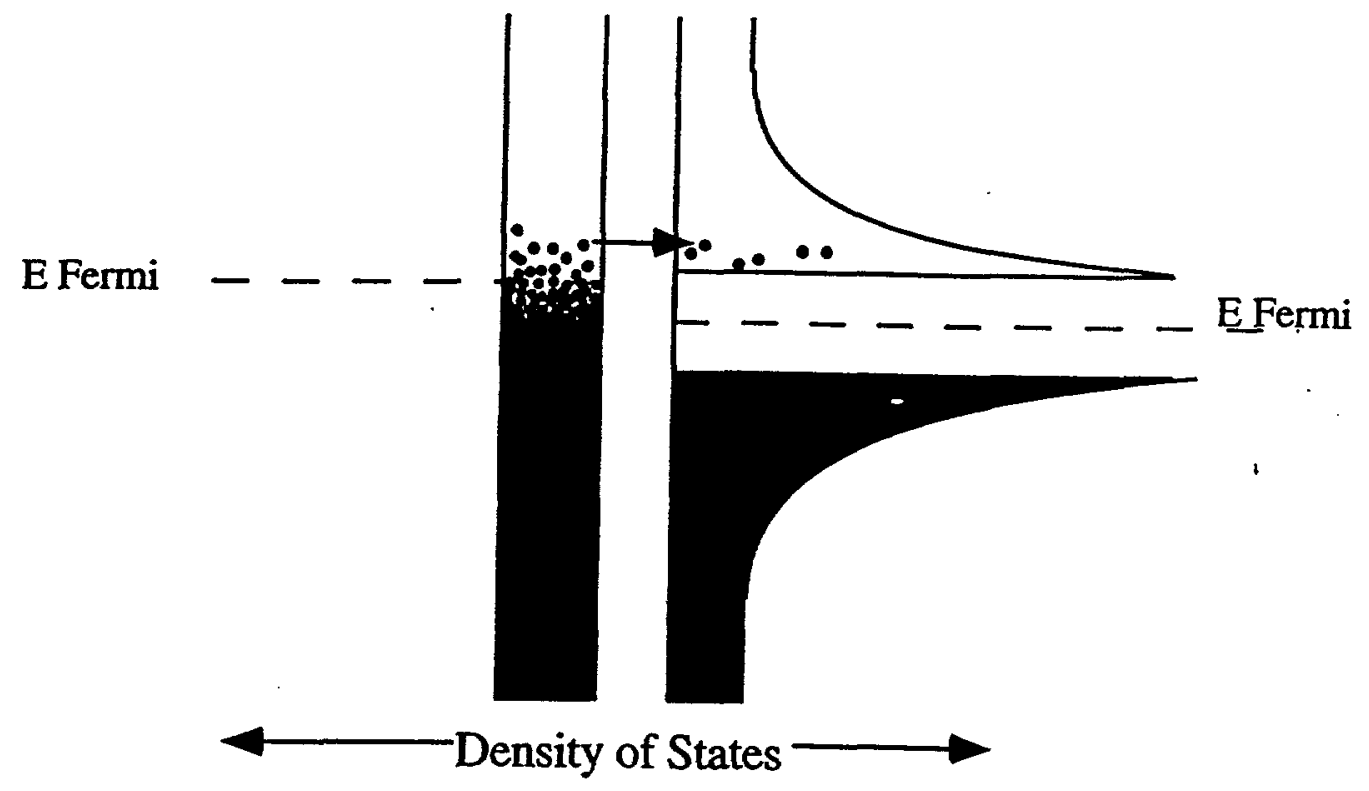

Figure 3.3. Tunneling diagram of an HET sensor. When biased at a voltage $\mathrm{Vb}$, only those electrons whose energy above the Fermi level is greater than $(\Delta-\mathrm{eVb})$ can tunnel, where $\Delta$ is the superconducting gap energy in the superconducting electrode. The number of such electrons is exponentially dependent on the temperature of the hot electrons, making the junction an excellent thermometer for the electron system. When energy in the form of quasipaticles enters the normal metal electrode, it is quickly thermalized via electron-electron and electron-phonon interactions, and the electron temperature is increased. At low temperatures, $T \leq 0.1 \mathrm{~K}$, the electrons are sufficiently decoupled from the phonon heat bath that they remain warm long enough (several microseconds) for their temperature to be accurately measured by noting the amplitude of the current pulse that the heating of these electrons produces. 


$$
\Delta E=2.35\left(k T^{2} C\right)^{1 / 2}\left[1+A\left(1+G_{e-p h} / G_{t u n}\right)\left(1+S_{i, a m p} / S_{i, t u n}\right)\right]^{1 / 2},
$$

where $T$ is the operating temperature, $C$ is the heat capacity of the electrons, $G_{e-p h}$ and $G_{\text {un }}$ are the thermal conductances due to phonon emission and tunneling. $S_{i, a m p}$ and $S_{i, t u n}$ are the spectral densities of the current fluctuations due to the amplifier and to the tunneling process, and $A$ is a bias-voltage-dependent factor of order unity. The best obtainable resolution is the thermodynamic limit, $2.35\left(\mathrm{kT}^{2} \mathrm{C}\right)^{1 / 2}$. Assuming that no electrons tunnel backward from the normal metal into the superconductor, this limit is approached when the tunneling current is large enough that the electrons cool predominately by tunneling, and the current is measured using a current amplifier with low noise, such as a superconducting quantum interference device (SQUID).

As a specific example, we estimate the performance of the test spectrometer we fabricated in the initial stage of this project. The absorber was a $0.2 \mu \mathrm{m}$-thick copper film $150 \mu \mathrm{m} \times 500 \mu \mathrm{m}$. At $0.1 \mathrm{~K}$, the thermodynamic limit for the resolution is about 2.0 $\mathrm{eV}$. Of course, the energy resolution of any detector system made using these sensors may be limited be other effects, such as the statistical nature of energy sharing between the various types of excitations, i.e. electrons and phonons. A simple calculation of this energy sharing effect also yields a resolution of about $2 \mathrm{eV}^{8}$. The extremely precise measurement of the energy absorbed by the normal electrons makes it possible to study these other effects systematically in order to reduce their influence.

\section{First experimental results -- Proof of Principle}

\section{A. Introduction}

In the first part of this project, we undertook a series of experiments to rapidly evaluate the feasibility of using this type of sensor in a cryogenic detector system. To do this we chose to fabricate test HET sensors by physical vapor deposition (evaporation) of metal films using mechanical shadow masks to define the device structure. Mechanical shadow masks were chosen because they allow us to evaluate a number of materials for suitability without having to learn and/or develop procedures to etch them controllably, as we would have if we had chosen to use photolithographic techniques. In addition, the shadow mask technique is more flexible in the geometry of structures that can be fabricated. The main disadvantage of this technique is that it is difficult to fabricate structures with features much smaller than $50 \mu \mathrm{m}$. Structures with such small features were not needed for these exploratory experiments. 


\section{B. Fabrication}

Test devices were fabricated in our existing high-vacuum evaporation system. In this system, a rotatable source assembly allows deposition from up to eight different resistively heated evaporation sources without exposure to air. A needle valve allows a controlled amount of ultra-pure oxygen to be admitted into the evaporator to oxidize metal films in order to create the thin insulating barrier through which the electrons tunnel in our devices. In order to control the position of the shadow mask as precisely as possible, we designed and built a precision mask manipulator. A photograph of this manipulator is shown in Figure 4.1. The position of the mask is controlled using precision micrometers. These micrometers allow a nominal positioning precision of 2.5 $\mu \mathrm{m}$. In actual practice repeatability was closer to $10 \mu \mathrm{m}$.

A schematic top view of the test sensor we fabricated is shown in Figure 4.2. The device consists of crossed-strips of superconducting and normal materials, and several superconducting wiring electrodes to provide electrical contact to the normal metal electrode. The device is fabricated as follows. First, the superconducting base electrode, made from $200 \mathrm{~nm}$ of aluminum is deposited. The sample is then allowed to cool, and 2 Torr of oxygen is admitted to the vacuum chamber to form the insulating tunnel barrier via oxidation of the top few nanometers of the aluminum film. Then a strip made from $200 \mathrm{~nm}$ of copper (a normal metal) is evaporated across the counter electrode and barrier, forming the tunnel junction. Then aluminum wiring electrodes are evaporated as shown, providing multiple electrical contacts to the normal metal electrode. These contacts allow us to bias the junction by applying a voltage, a then to measure both the steady state and X-ray induced current. Note that in this design there is no separate superconducting absorber. The idea of this experiment was to test the response of the sensor alone, unfettered by additional absorbing elements, by absorbing $x$-rays directly in the normal metal electrode of the sensor.

\section{I-V curves}

The first measurements we did was to measure the current-voltage (I-V) characteristics of the junctions to assess the quality of the tunnel barrier. If the tunnel barrier is of insufficient quality, resistive leakage current would cause excess noise and heating, and make it impossible to operate the device as a high resolution energy sensor.

The theoretical I-V curve for an ideal NIS tunnel junction with a normal state resistance of $1.0 \Omega$ is shown in Figure 4.3. At zero temperature, when the applied voltage $V$ is less than the gap voltage $\Delta / e$, no current can flow, since the Fermi level in the normal electrode is below the level of the lowest available state in the superconducting electrode. Then, as the voltage is raised above the gap voltage, a rapid increase in tunneling current occurs as occupied states below the Fermi level in the normal metal electrode line up with empty states in the superconducting electrode. As the temperature increases from 


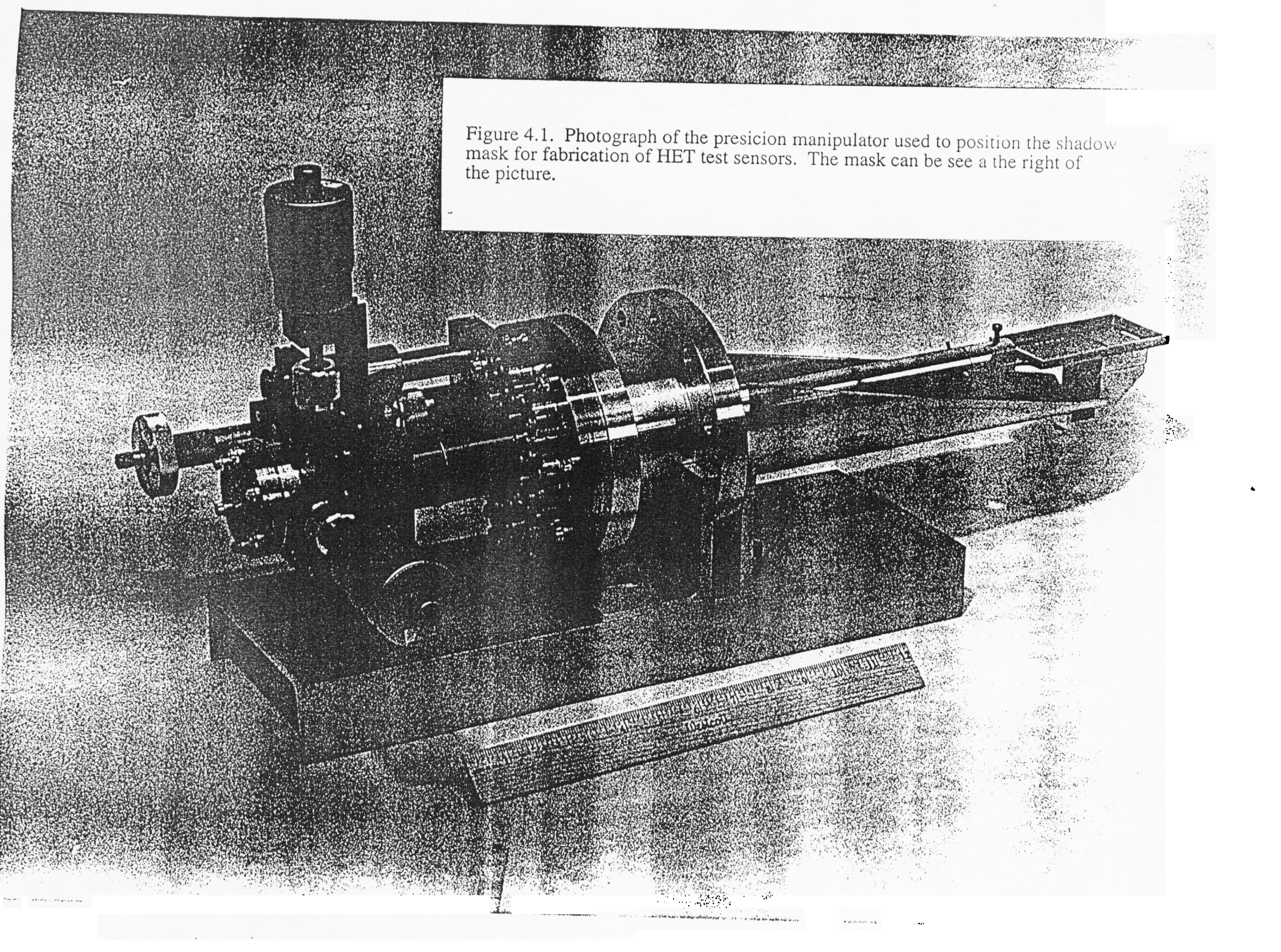




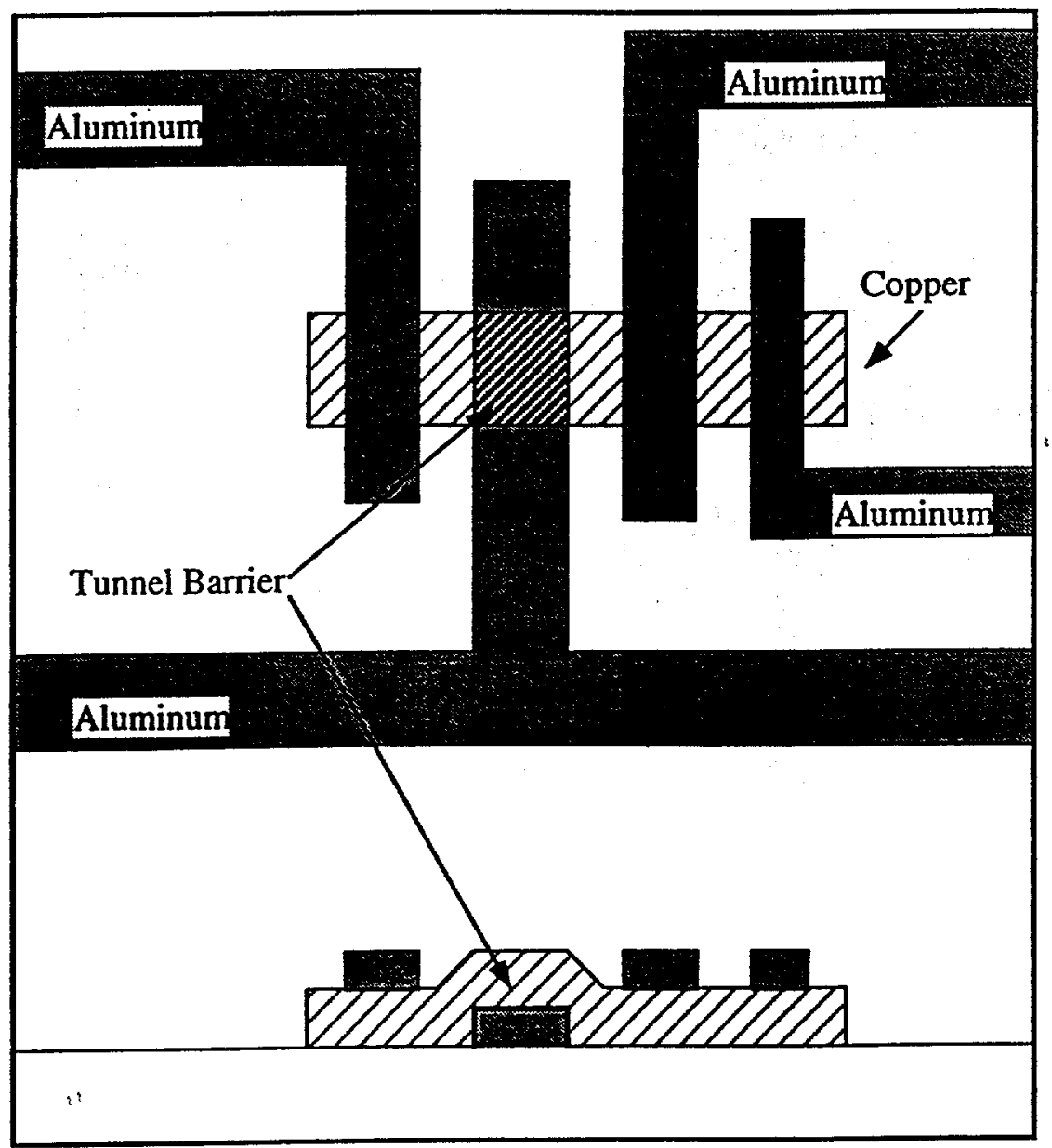

Figure 4.2. Schematic diagram of the HET test sensor. The tunnel junction is formed by crossed strips of superconducting and normal metals with a thin insulating barrier in between them. Aluminum wires serve to provide electrical contact to the junction. 


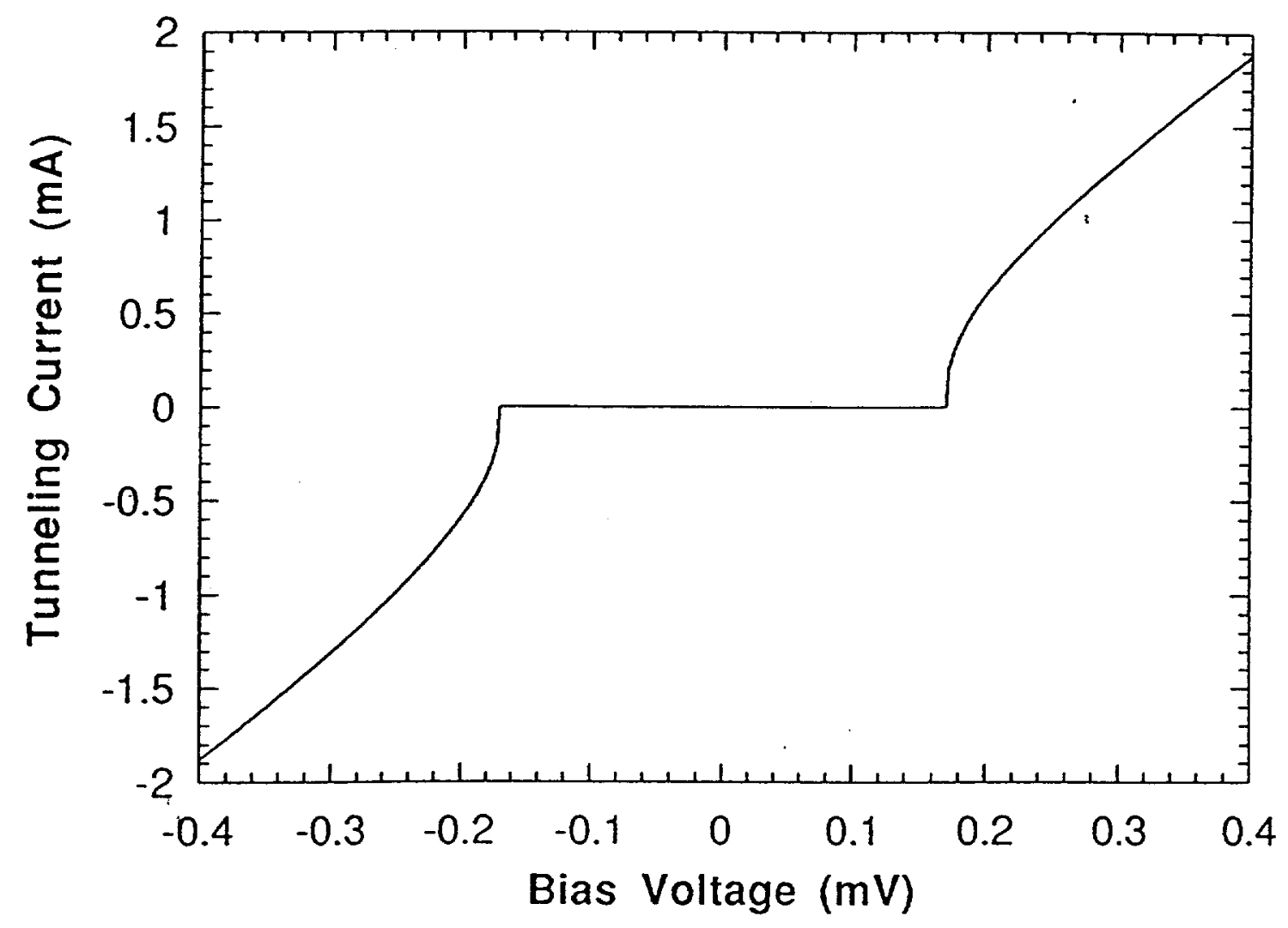

Figure 4.3. Theoretical I-V curve of a NIS tunnel junction. The parameters of the calculation were: $R_{n}=0.2 \Omega, T=0.06 \mathrm{~K}, \Delta=0.17 \mathrm{meV}$. Note the sharp feature at the gap voltage $\mathrm{V}=0.17 \mathrm{mV}$. 
zero, more and more electrons are excited to states above the Fermi level, leading to the increasing current in the region below the gap voltage.

A typical I-V curve of one of our test sensors measured at $62 \mathrm{mK}$ is shown in Figure 4.4. the shape if the I-V curve is qualitatively similar to the expected shape, but differs in two ways. First, there is a small amount of resistive leakage current at voltages significantly below the gap voltage. The magnitude of the leakage current is low enough (less than $10^{-3}$ of the normal state current), that it will not adversely affect the operation of the sensor. The second difference is more important. Notice that the region of the curve just below the gap voltage is more rounded than would be expected for a NIS junction measured at this temperature. This is an indication that the biased NIS junction may not be getting as cold as expected, due to self heating from the bias current. We will provide more evidence for this self heating in the next subsection, and discuss it in more detail in section $\mathrm{V}$.

\section{Energy measurements with X-Rays}

To measure the performance of the device as an energy sensor, we measured its response when X-rays were absorbed in the normal metal electrode. This provides a nearly instantaneous deposition of a know amount of energy, making it an ideal method for characterizing the response, noise properties, and energy resolution of the sensor.

Because of the low output impedance of the sensor, it is poorly matched to existing FETbased current amplifiers. In order to measure the $\mathrm{X}$-ray induced current pulse with high precision, we used a novel current amplifier based on Superconducting Quantum Interference Devices (SQUIDs). This amplifier uses a series array of 100 SQUIDs magnetically coupled to a single input coil ${ }^{12}$. The use of the series array boosts the output impedance of the SQUID by a factor of 100 , providing a good match to roomtemperature amplifiers based on bipolar transistors. Although this amplifier is still under development, we were able to obtain several sample amplifiers from the manufacturer. The design of these amplifiers is still not optimized -- as such, the noise performance of these test amplifiers was more than a factor of ten worse that what will ultimately be realized. All X-ray measurements discussed in this report were done using these amplifiers, and hence, the contribution to the total noise due to these amplifiers is expected to improve markedly in the near future.

We exposed a test sensors to X-rays from an ${ }^{55} \mathrm{Fe}$ source which emits $\mathrm{Mn} \mathrm{K \alpha}$ and $\mathrm{Mn}$ $\mathrm{K} \beta \mathrm{X}$-rays at $5.89 \mathrm{keV}$ and $6.49 \mathrm{keV}$. A typical pulse produced when a Mn Ka X-ray is absorbed is shown in Figure 4.5. The pulse has an amplitude of $0.4 \mu \mathrm{A}$, and decays exponentially with a time constant of $3.5 \mu \mathrm{s}$. The decay time is determined by the cooling time of the hot electrons in the normal metal of the sensor. If we make a histogram of pulse heights, we should produce an energy spectrum of our ${ }^{55} \mathrm{Fe}$ source. Such a histogram is shown in Figure 4.6. At the right side of the spectrum, we are able to resolve the $\mathrm{Mn} \mathrm{Ka}$ and $\mathrm{Kb}$ lines as indicated. The counts in these two define line result from X-ray absorption events that occur directly over the tunneling region. Other counts at lower pulse height are due to absorption events elsewhere along the normal metal strip. 


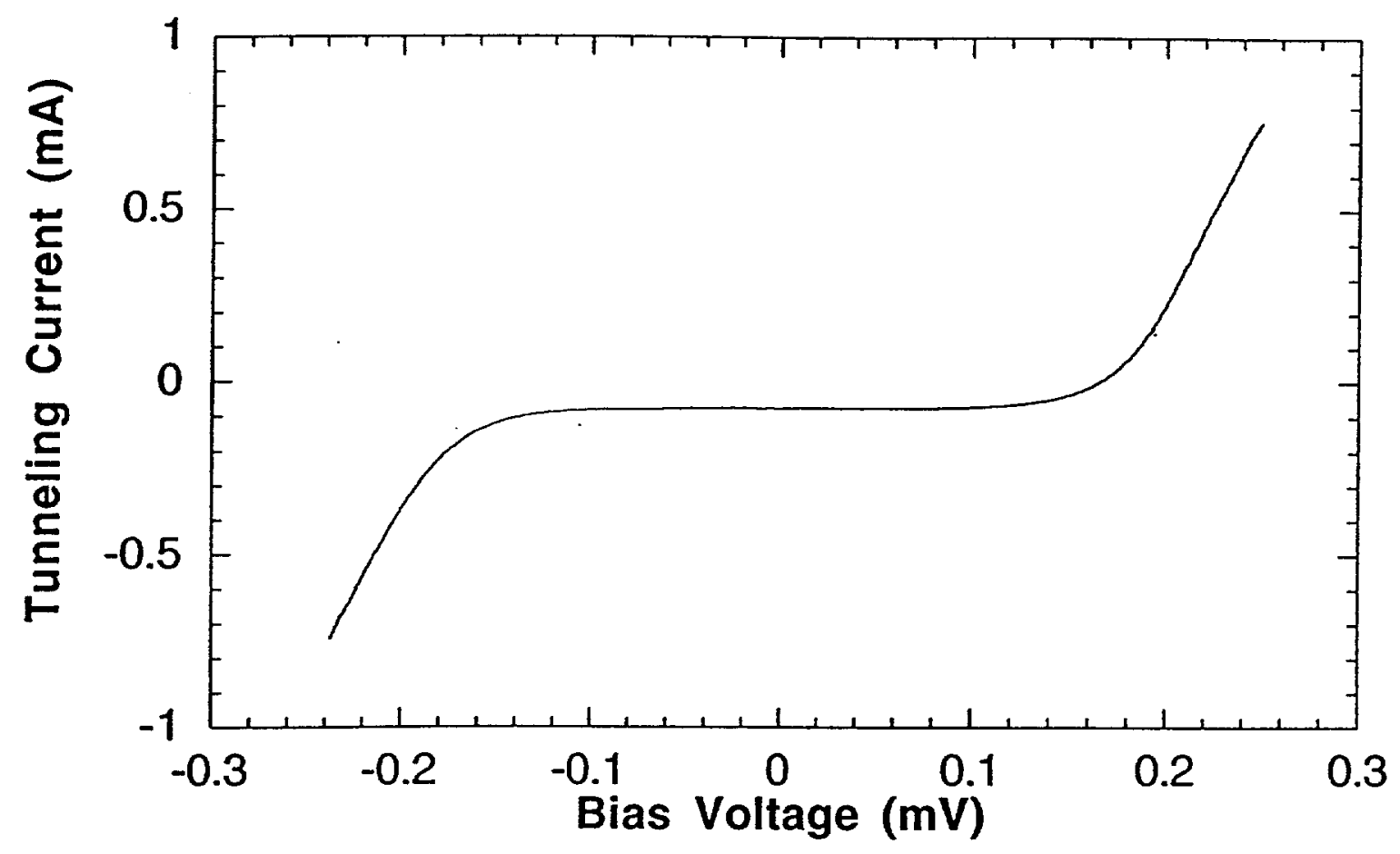

Figure 4.4. I-V curve of a test sensor measured at a temperature of $63 \mathrm{mK}$. The shape is qualitative similar to the expected shape shown in Figure 4.3, but differs in two ways. First, there is a small amount of resistive leakage at low bias voltage. Second, the current rise just below the gap voltage is more rounded than expected, leading us to suspect biasinduced self heating in this device. 


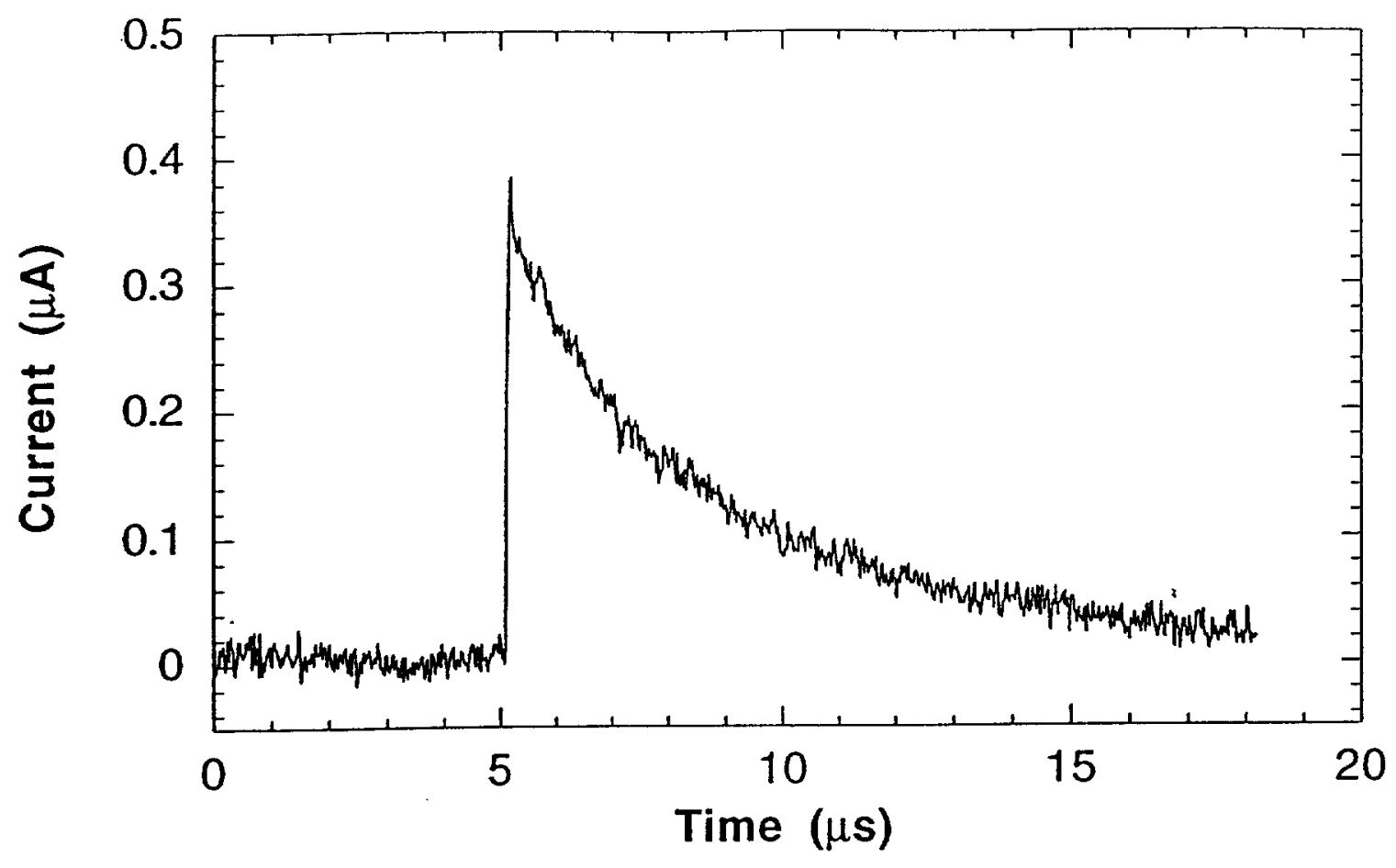

Figure 4.5. Current pulse measured when a $6 \mathrm{keV} \mathrm{X-ray} \mathrm{was} \mathrm{absorbed} \mathrm{inthe} \mathrm{test} \mathrm{sensor.} \mathrm{The}$ pulse was measured at a temperature of $100 \mathrm{mK}$ using a high-speed current amplifier based on an array of SQUDs. 


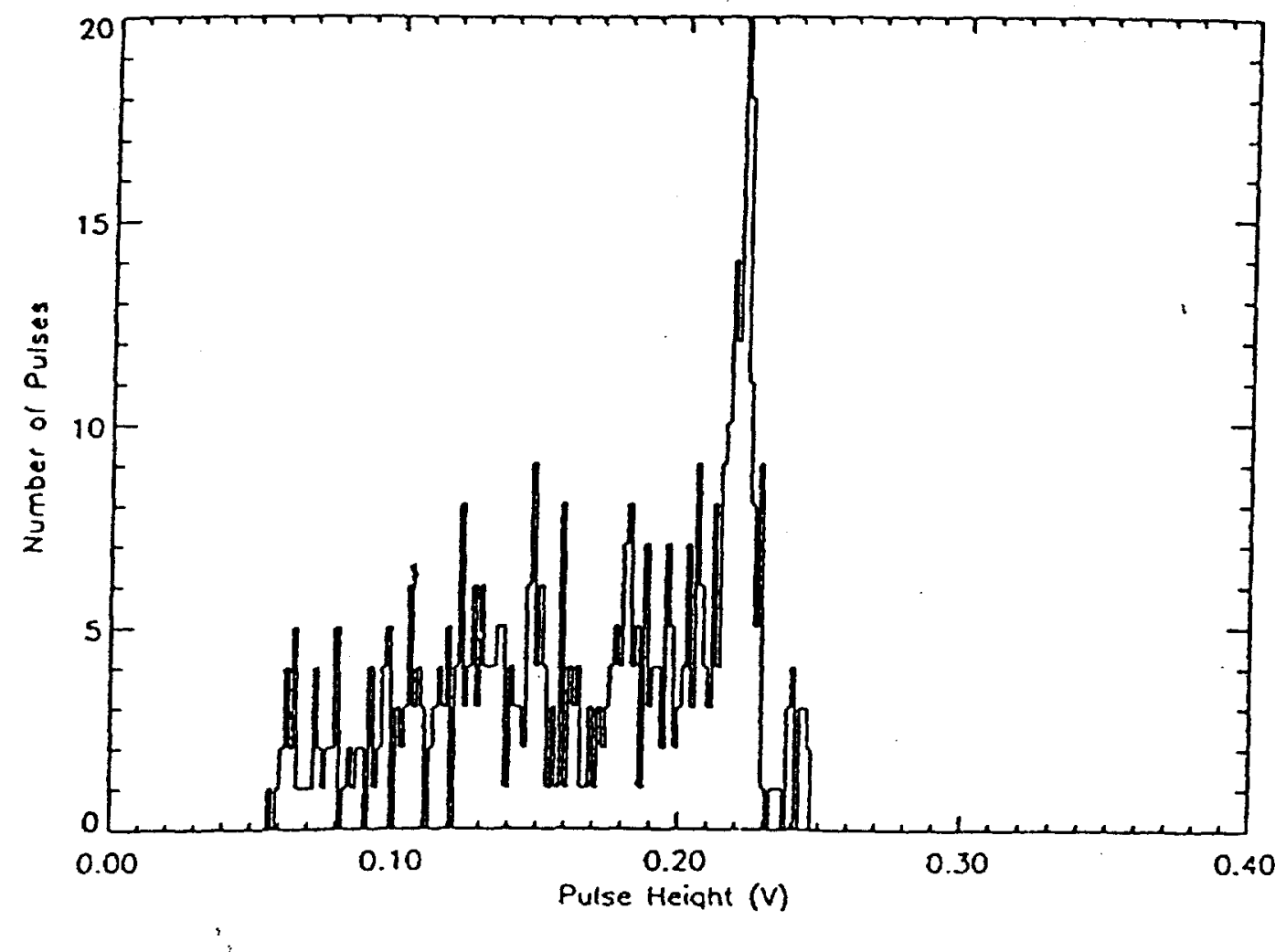

Figure 4.6. Typical spectrum measured when our test sensor was exposed to radiation from an $55 \mathrm{Fe}$ source, which emits $\mathrm{Mn} \mathrm{Ka}$ and $\mathrm{Kb}$ X-rays at $5.89 \mathrm{keV}$ and $6.49 \mathrm{keV}$. We are able to resolve these two lines as shown. Counts in these two lines result from X-ray absorbtion events that occur directly over the tunnel region. Counts at lower pulse height arise from $\mathrm{X}$-rays that are absorbed elsewhat in the normal metal strip. 
This position dependence occurs because the electrons begin to cool before they uniformly fill the length of the normal metal strip. This effect is exacerbated by the shorter-than-expected decay time of the hot electrons, and the short mean free path in our normal metal film. It can be reduced by decreasing the size of the normal metal electrode, reducing the number of impurities in the normal metal film, or by understanding and eliminating the cause of the short decay times.

To help understand the origin of the short decay times of the hot electron distribution, we plot the decay time of the current pulse as a function of temperature. Such a plot is shown in Figure 4.7, along with a theoretical estimate calculated using the standard theory of electron-phonon coupling. Note that at low temperatures, the decay time is much fast than we expect, indicating that the sensor may not be as cold as the bath temperature. This, along with the non-ideal I-V characteristic, is a strong indication that the sensor is not cooling due to excessive heating related to the bias current. Heating and cooling due to tunneling currents is discussed in detail in the next section.

\section{Heating and Cooling in the NIS tunnel junction}

\section{A. Introduction}

When we started work on the HET sensor, we expected that the normal electrode of the NIS tunnel junction would be cooled by tunneling currents ${ }^{13}$. Under closer examination of the flow of energy to and from the normal electrode during tunneling, we found this to be true only when there are sufficiently few quasiparticles present in the superconducting electrode so that the contribution to the energy flow due to electrons tunneling from the superconducting electrode back into the normal electrode is small.. First we review the naive picture of cooling, and then discuss the heating due to back-tunneling quasiparticles.

\section{B. Cooling}

In Figure 5.1, we show a schematic diagram of the forward tunneling process in an NIS tunnel junction. In this diagram, the junction is bias at a voltage $\mathrm{V}_{\mathrm{b}}$ just below the gap voltage $\Delta \mathrm{e}$. Under these conditions, only electrons with energies as least $\Delta \mathrm{e}-\mathrm{V}_{\mathrm{b}}$ above the Fermi energy are allowed to tunnel. When they tunnel, they remove this excess energy from the electron system, thus cooling the system ${ }^{13}$. To preserve charge neutrality, each tunneling electron is replaced by an electron at the Fermi surface from the wiring of the bias circuit, in our case, the superconducting wires attached to the normal metal electrode. For this reason, we initially expected our normal electrodes to be cooled by the tunneling process.

This cooling effect had been confirmed in an experiment conducted using sub-micron length scale NIS tunnel junctions ${ }^{13}$. In these small junctions, the quasiparticle created by the tunneling electron could rapidly diffuse away from the tunneling barrier into a normal 


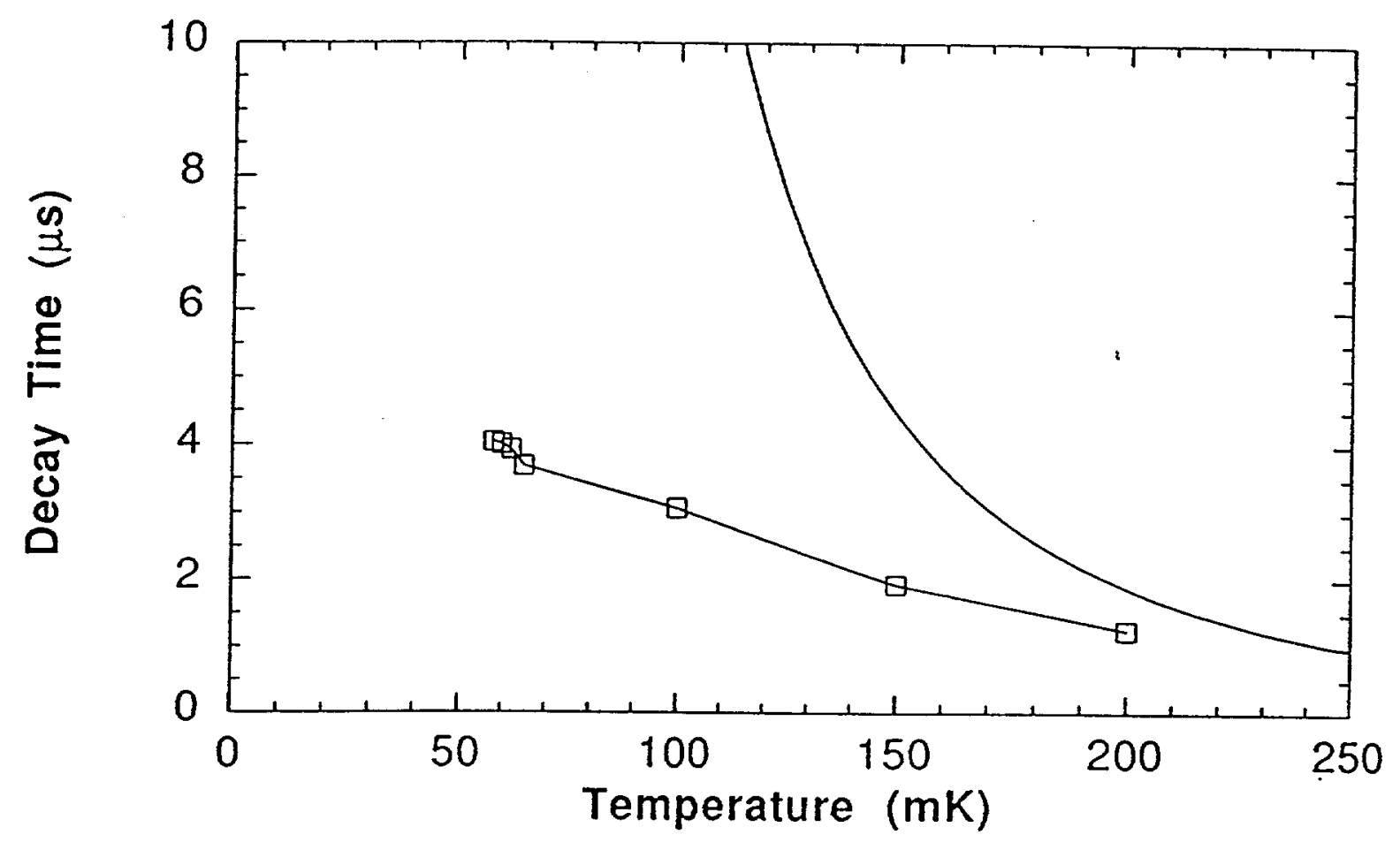

Figure 4.7. Pulse decay time as a function of bath temperature. The solid line is a theoretical estimate calculated using only electron-phonon coupling in the normal metal. The much reduced decay that we measure indicates that the sensor may be self-heating due to the applied bias power. 


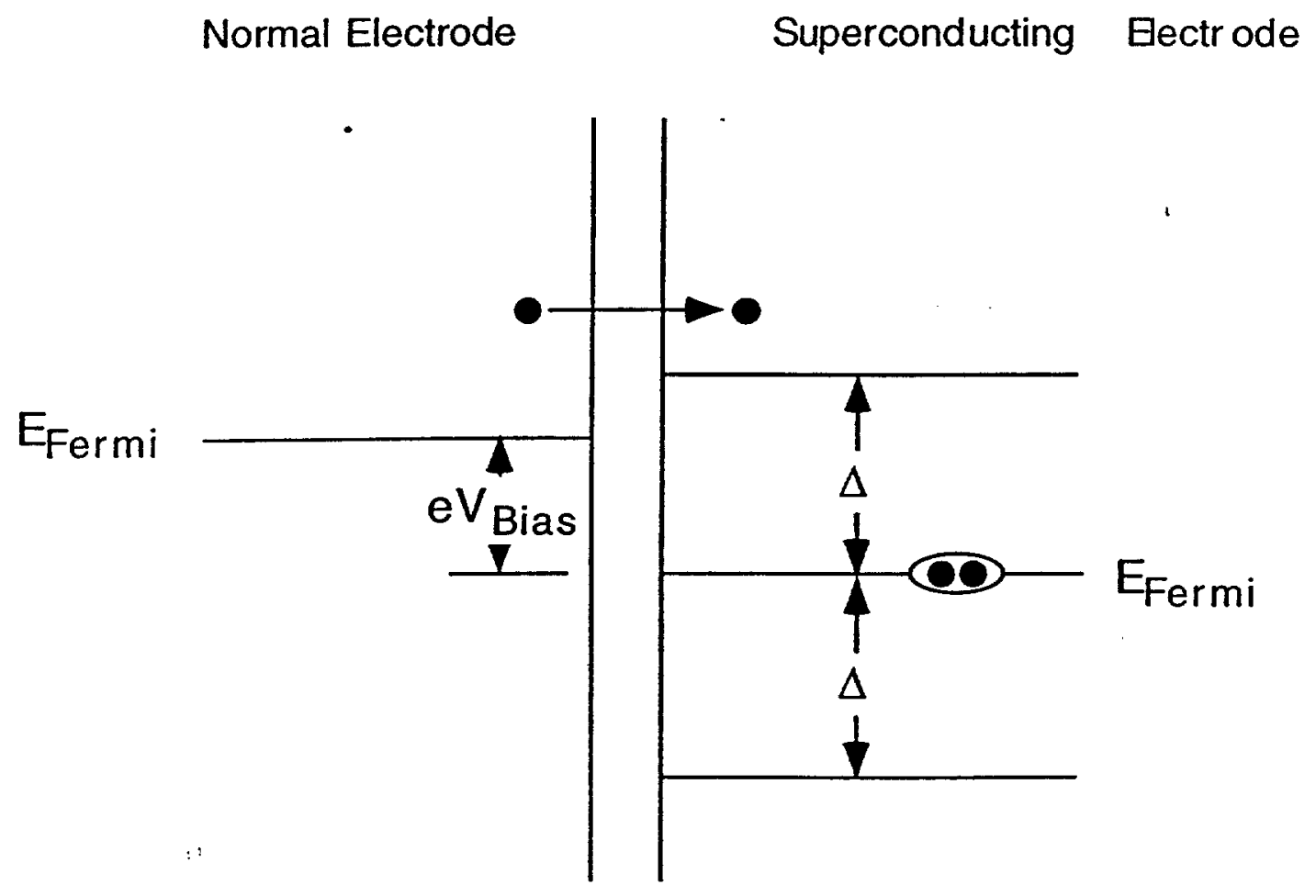

Figure 5.1. Schematic diagram of the forward tunneling process. Since only electrons whose energy is significantly above the Fermi eneryg are allowed to tunnel, this process cools the normal electrode. 
metal trap, so there was little or no probability for it to tunnel back into the normal metal. In our large junctions, this was no longer true, and there was significant probability that the quasiparticles would tunnel back into the normal electrode. In the next subsection, we discuss the energy flow due to these back-tunneling particles.

\section{Back Tunneling and Heating}

In figure 5.2, we show a schematic diagram of the two possible back tunneling processes that may occur to a quasiparticlè created by a forward tunneling event ${ }^{14}$. In the process marked A, the quasiparticle tunnels backwards into a state at the same energy it had when it left. This process simple replaces the energy lost what the particle tunneled the first time, and as such is unlikely to produce much excess heating. In process $B$, the electronlike quasiparticle created by the forward tunneling process tunnels backwards as a holelike quasiparticle. This is possible because the quasiparticle states with energies near the gap energy are superpositions of electron-like and hole-like states. What is important to note is that this process results in a state far from the Fermi surface -- a state that has an energy $\left(\Delta+\mathrm{eV}_{\mathrm{b}}\right)$, typically $10-20$ times the energy than the $\left(\Delta-\mathrm{eV}_{\mathrm{b}}\right)$ removed by the original tunneling event. If these processes are not highly suppressed, the heating due to these processes will exceed the cooling effect of the forward processes.

\section{Optimizing the Sensor Geometry to Reduce Heating}

\section{A. Introduction}

In the last section we saw that it is important to prevent the forward-tunneling-produced quasiparticles from tunneling backwards as holes. The only ways to prevent the back tunneling is to remove the quasiparticles from the tunneling region as fast as possible. This can be achieved in two ways. The first way is to place a normal metal trap directly under the superconducting electrode. In this case the quasiparticles need only to diffuse the several hundred nanometer thickness of the thin film that makes up the superconducting electrode to be trapped in the normal electrode away from the tunnel barrier. The second way is to place a normal metal trap in contact with the superconducting electrode, but to the side of the junction. In this case, the quasiparticles must diffuse horizontally to reach the trap. Numerical modeling indicates that the horizontal dimension of the tunnel junction must be less than $\sim 10-20 \mu \mathrm{m}$ for this process to be efficient enough for typical junction and film parameters. Thus this second approach requires shrinking of the junction feature size to less than that which is readily obtainable using our shadow mask technology. 


\section{Normal Electrode Superconducting Eectr ode}

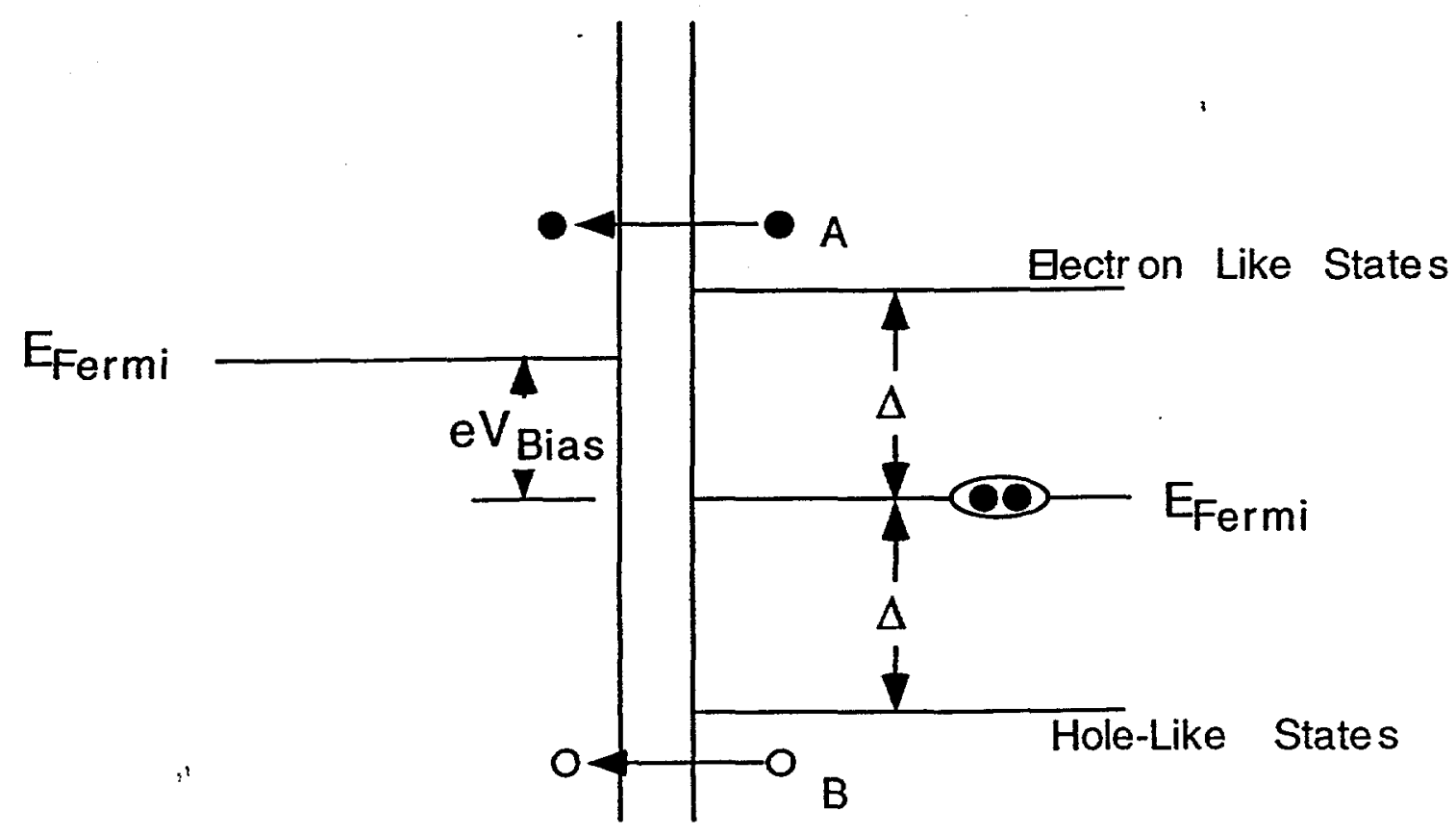

Figure 5.2. Schematic diagram of the two reverse tunneling processes. The top process is simply the reverse of the process hsown in Figure 5.1. In the bottom process, the quasiparticle in the superconductor has flipped across the Fermi level to become a holelike state. This is possible because quasiparticle states near the gap edge are superpositions of hole-like and electron-like states. Note that this process leaves a hole deep below the Fermi energy in the normal metal. When electrons relax to fill this state, a considerable amount of energy is released, heating the normal metal electrode. 
Top View

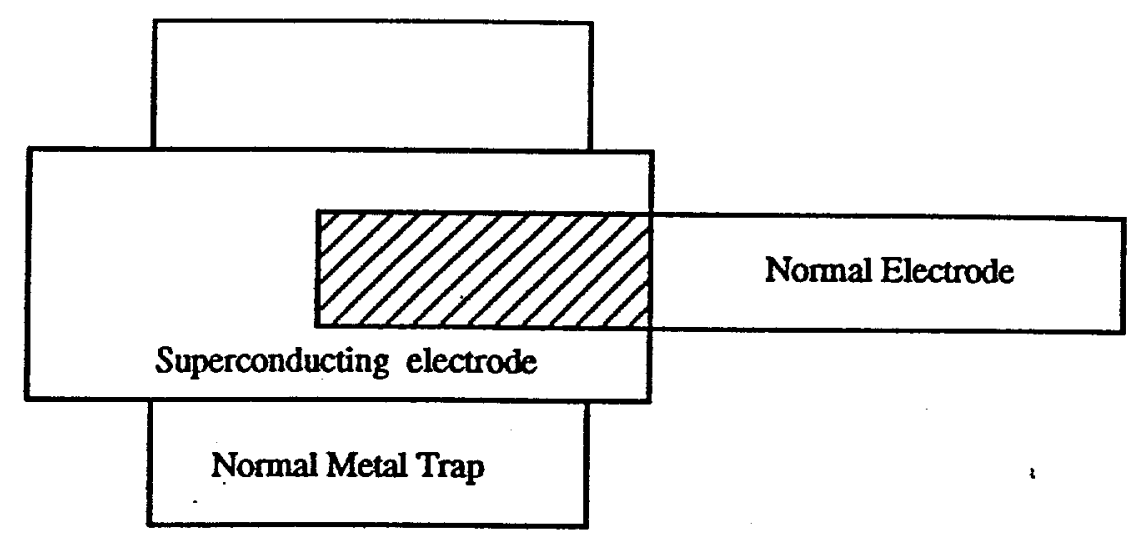

Side View

Tunnel Barrier

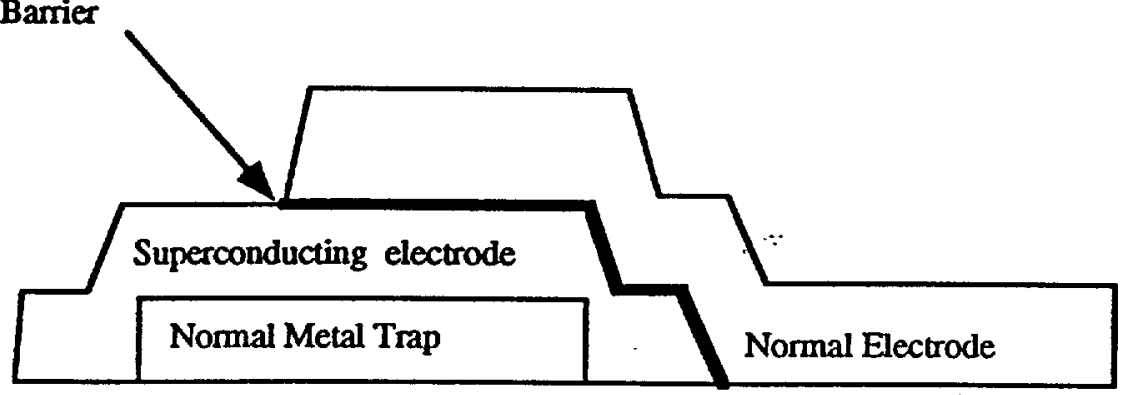

Figure 6.1 Schemetic diagram of an HET sensor with a normal metal trap directly under the tunneling region. 


\section{B. Trap directly under junction}

We have designed and tested sensors to try both approaches. First we discuss the approach of placing the trap directly under the junction area. We designed a new set of shadow masks that produces a device shown schematically in Figure 6.1. In this device, a normal metal trap made by depositing a $200 \mathrm{~nm}$-thick film of silver directly beneath most of the tunneling region. We cannot extend the trap to the region at the extreme right of the tunneling area without causing a very leaky junction due to normal-insulatornormal NIN tunneling directly from the trap to the normal metal electrode. The remainder of the junction is fabricated in a fashion similar to the devices discussed in section IV. At the same time, on the same substrate, we also fabricate a junction identical in design except that it does not have the additional normal metal trap. This allows a direct comparison to assess the effects of including this trap.

In Figure 6.2, we plot typical I-V curves measured from devices with and without this trapping layer. The first feature we notice is that in the case of the junction with trap, the onset of tunneling current occurs at much lower voltage than it does for the trapless junction. This is because the contact of the superconducting electrode to the normal electrode lowers the gap in the normal electrode via the proximity effect. The normal metal under the superconductor is also likely to be driven superconducting by this same effect. It is possible that the proximity effect smears the energy gap in the superconducting electrode. If this is the case, excess current just below the current rise at the gap energy might be due to this gap smearing. Thus, it is impossible gain significant information conceming the temperature of the normal metal in the junction by evaluating the $\mathrm{I}-\mathrm{V}$ curve. Instead we must measure the change in pulse characteristics with bias voltage.

In Figure 6.3, we plot the decay time of $\mathrm{X}$-ray induced pulses as a function of temperature for several different bias voltages. We plot the data for both the junction with a normal metal trap, and for the junction with no trap. While there are some differences between the two junctions, they exhibit qualitatively similar behavior. The decay time of both junctions saturates at low temperature, rather than continuing to increase as the temperature is lowered. This indicates that the junction is still not cooling efficiently and that this approach to cooling the junction is not effective. This could be due to two factors. First, due to the proximity effect, the energy gap in the trap material is not very much lower that in the superconducting electrode. Since the trapping speed varies as the cube of the gap difference, this small difference reduces the efficiency of the trapping process dramatically. Second, when the particles are trapped, or quasiparticle pair in the trap recombine to form Cooper pairs, phonons are emitted. If these phonons travel back into the normal metal and are absorbed, they can heat the normal metal. We estimate that between 10 and 25 percent of the energy emitted into phonons is absorbed in the normal metal electrode. The heating in the device with the trap could be due to either of these two factors. Since this approach prove to be unsuccessful, we moved on to try the second approach of having the normal metal trap to the side of the junction. 

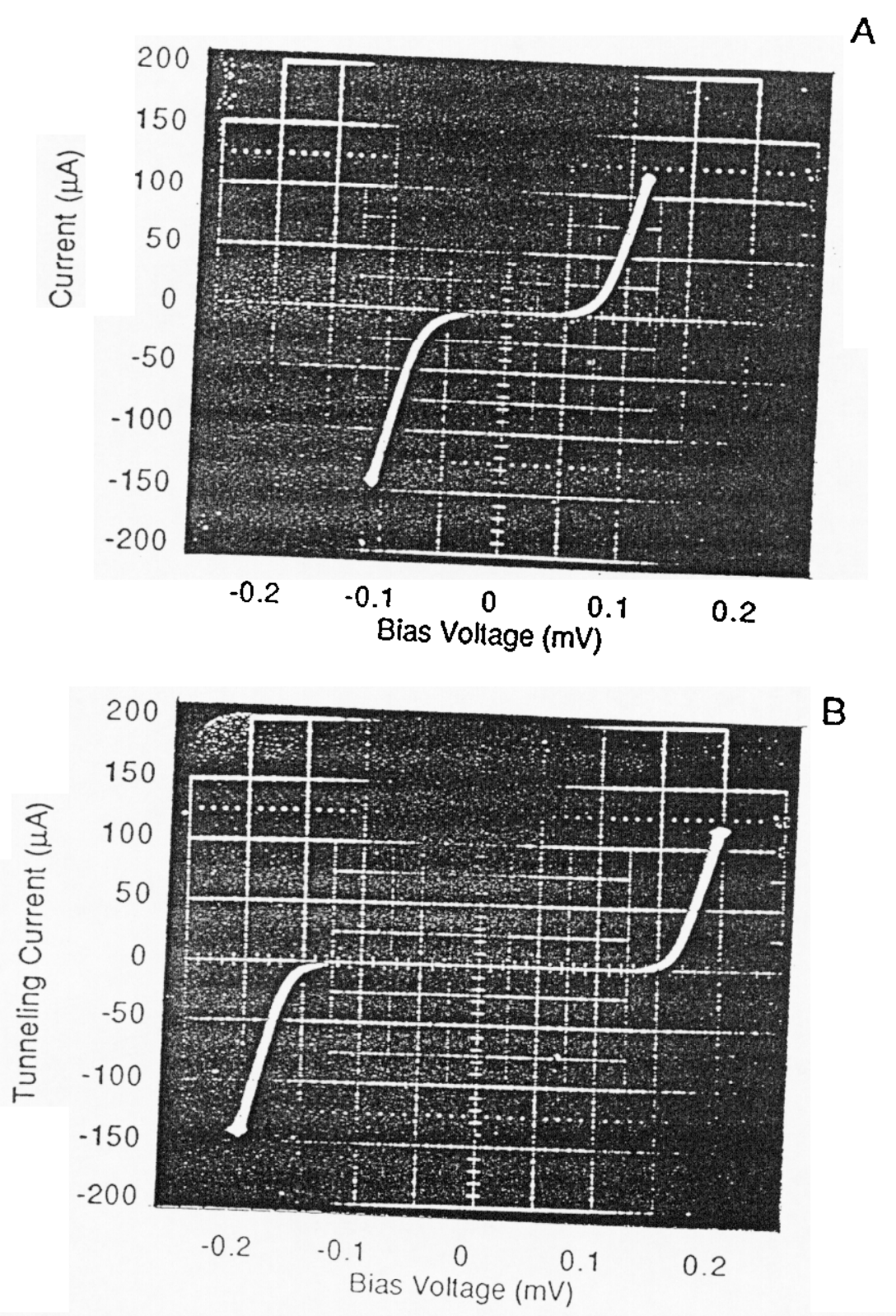

Figure 6.2. I-V curves of junctions with (A) and without (B) normal metal trapping layers. Note the reduced gap voltage for the junction with the trapping layer. This is due 10 a reduction of the gap in the aluminum due to the proximity effect. 
With Normal Metal Trap

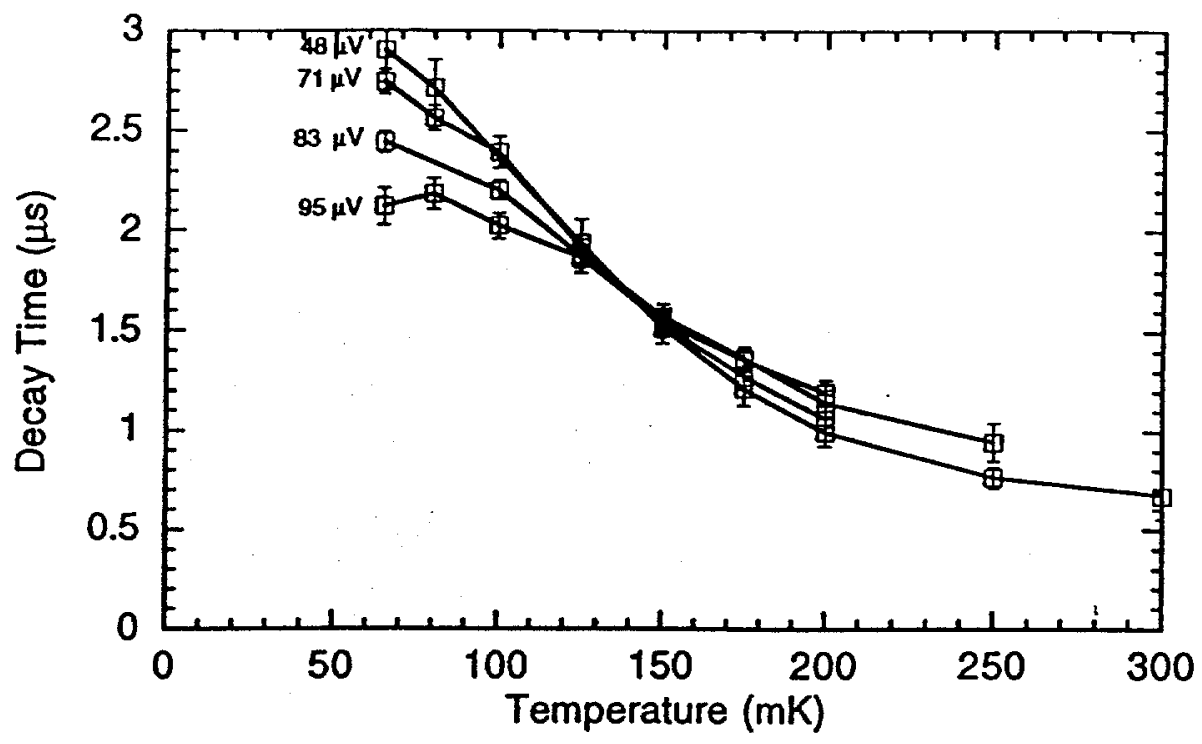

Without Normal Metal Trap

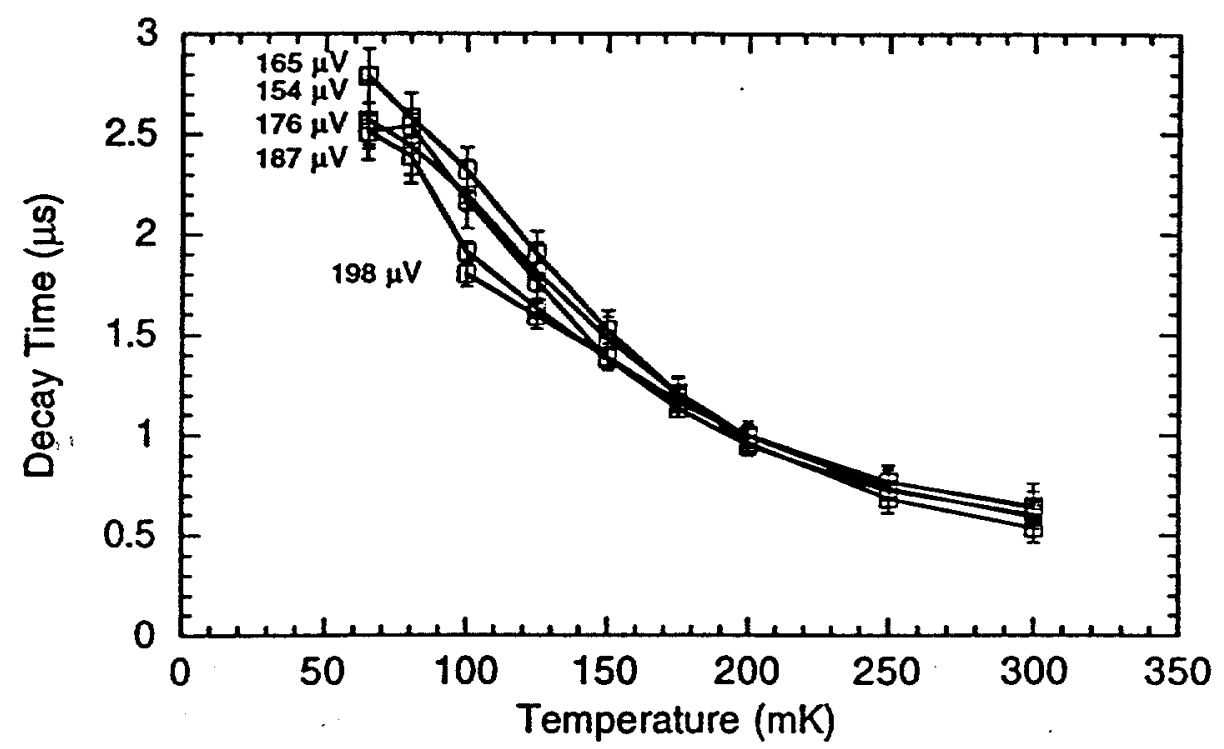

Figure 6.3. Decay time as a function of bath temperature for test sensors with and without a normal metal trap directly beneath the tunneling area. The decay time was measured at several different bias voltage for both devices. There is no significant difference between the two devices, indicating that this scheme did not significantly cool the junctions. 


\section{Trap to the side of the junction}

When the quasiparticle trap is to the side of the junction, the junction must be small enough to allow quasiparticles created near the center of the junction to diffuse rapidly to the trap before they undergo a back tunneling event. For this to be the case, we estimate that the junction should be less than $10-20 \mu \mathrm{m}$ across. Junctions of this small size cannot be easily fabricated using our shadow mask technique, and must be fabricated using photolithography. Instead of developing our own photolithographic process; we chose to collaborate with Barbara Neuhauser's group at San Francisco State University, where a photolithographic process to produce high-quality NIS tunnel junctions was already under development.

In this process, aluminum, aluminum oxide, and cooper layers are deposited over the entire surface of a silicon wafer, in effect making a huge junction. Subsequent etching steps remove material over most of the area, defining the junctions to be studied. Top and cross-sectional views of junctions made using this process are shown in Figure 6.4. The use of photolithography allows the fabrication of NIS junctions with linear dimensions as small as $5 \mu \mathrm{m}$, small enough for the quasiparticles to rapidly diffuse away from the tunneling region.

The first junctions made with this process were $100 \mu \mathrm{m} \times 100 \mu \mathrm{m}$. The decay times of $\mathrm{X}$-ray induced pulses in these large sized junctions were studied and it was found that these junction to appeared to suffer from self heating. Only very recently have junctions with smaller linear dimensions been fabricated. A series of devices, all with the same tunneling area, normal resistance, and normal metal volume, but with different length to width ratios have been produced. Using these junctions, it will be possible to test the affect of the diffusion of quasiparticles away from the tunneling area on device performance. Very preliminary results gathered by measuring I-V characteristics indicate that junctions with very high length to width ratios $(1000 \mu \mathrm{m} \times 10 \mu \mathrm{m})$ may cool more efficiently that square junctions with the same area.

\section{1-D X-ray imagers}

\section{A. Introduction}

Although we have not yet reached the predicted resolution for the HET sensor, we have analyzed the operation of these devices, and gained significant insight into the physics of their operation. While their operation is complicated by unforseen heating behavior, we are confident that this can be overcome. Encouraged by this understanding, we have begun to develop ways to incorporate these sensors into X-ray and gamma-ray spectrometers with enough area and/or volume to be useful to the scientific and technical community. In this section, we discuss our efforts to fabricate high resolution X-ray spectrometers with one-dimensional imaging capability along the long direction of a 900 


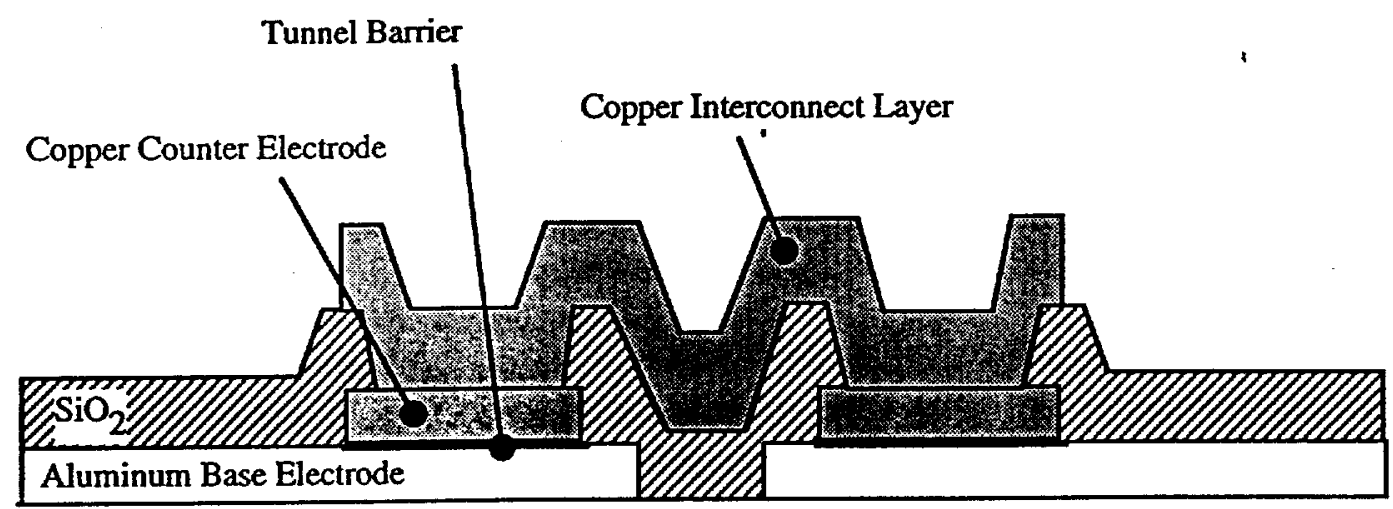

Figure 6.4. Schematic cross section of a photolithographically defined test sensor. The aluminum base electrode and the copper counter electrode form a series pair of NIS tunnel junctions. Junction dimensions can be as small as $10 \mu \mathrm{m}$. 
$\mu \mathrm{m} \times 300$ absorber strip. In Section VIII, we discuss our plans for using HET sensors in gamma-ray spectrometers based on high-purity superconducting crystals.

\section{B. Detector concept, design and fabrication}

As we have seen previously, finite diffusion speed, coupled with relaxation effects, causes significant dependence of the pulse height on the position of the absorption event. For thin film absorbers larger than $-100 \mu \mathrm{m}$, this effect degrades the energy resolution of the spectrometer to an unacceptable level. There are two ways around this problem. The first is to design spectrometers so that the quasiparticles in the absorber only need to diffuse $50-100 \mu \mathrm{m}$ before they tunnel. While this is a viable approach in some cases, there are many instances where one would want a spectrometer with a larger active area than this approach allows. The second approach is to use two or more sensors attached to a single absorber to measure and account for this position dependence. This has the added advantage that one also obtains the location of the absorption event, and thus with appropriate optics, the detector is imaging. The simplest realization of this type of spectrometer is a narrow strip of absorbing material with sensors on either end. By measuring the amount of energy that arrives at the two sensors, we can infer both the location of the $\mathrm{x}$-ray absorption event along the strip, and the energy of the $\mathrm{X}$-ray photon that was absorbed. This type of detector has already been demonstrated using SIS sensors $^{6}$. We attempted to use our HET sensors for this type of spectrometer.

A diagram of the imaging spectrometer is shown in Figure 7.1. X-rays are absorbed in the $300 \mu \mathrm{m}$ wide by $900 \mu \mathrm{m}$ long film made from lead. Lead is an efficient absorber of $\mathrm{X}$-rays. The $\mathrm{X}$-ray induced quasiparticles diffuse into the normal metal electrodes if the HET sensors, where the energy that arrives in each sensor is precisely measured. Then the absorption location and energy deposited is inferred. In order to reduce energy loss due to high energy phonon escaping into the substrate, the lead absorber is fabricated on top of a thin (200 nm-thick) membrane made from silicon nitride. The structure of all films is defined by shadow-mask lithography of films deposited by physical vapor deposition (evaporation). The junction is formed by deposition of a $200 \mathrm{~nm}$ silver normal metal electrode on top of the oxidized surface of the $200 \mathrm{~nm}$-thick aluminum base electrode. Then the $500 \mathrm{~nm}$-thick lead absorber is deposited followed by the aluminum wiring traces. All steps after the oxidation of the base electrode were done without exposing the sample to air, so the interfaces between the absorber and the sensors should be clean and free of impurities.

The I-V curves of the junctions that make up these devices indicated that the junctions were of high quality. When exposed to $\mathrm{X}$-rays from an ${ }^{55} \mathrm{Fe}$ source, the devices produced a variety of pulses with different shapes and heights, each presumably arising from a different region of the detector. In order to understand the origin of the various types of pulses, we digitize a large number of pulses an make a scatter plot of the rise time of each pulse as a function of its height. An example of such a plot is shown in Figure 7.2. In this plot, dots that correspond to pulses due to the absorption of X-ray photons in different regions of the absorber appear in different regions of the diagram. 


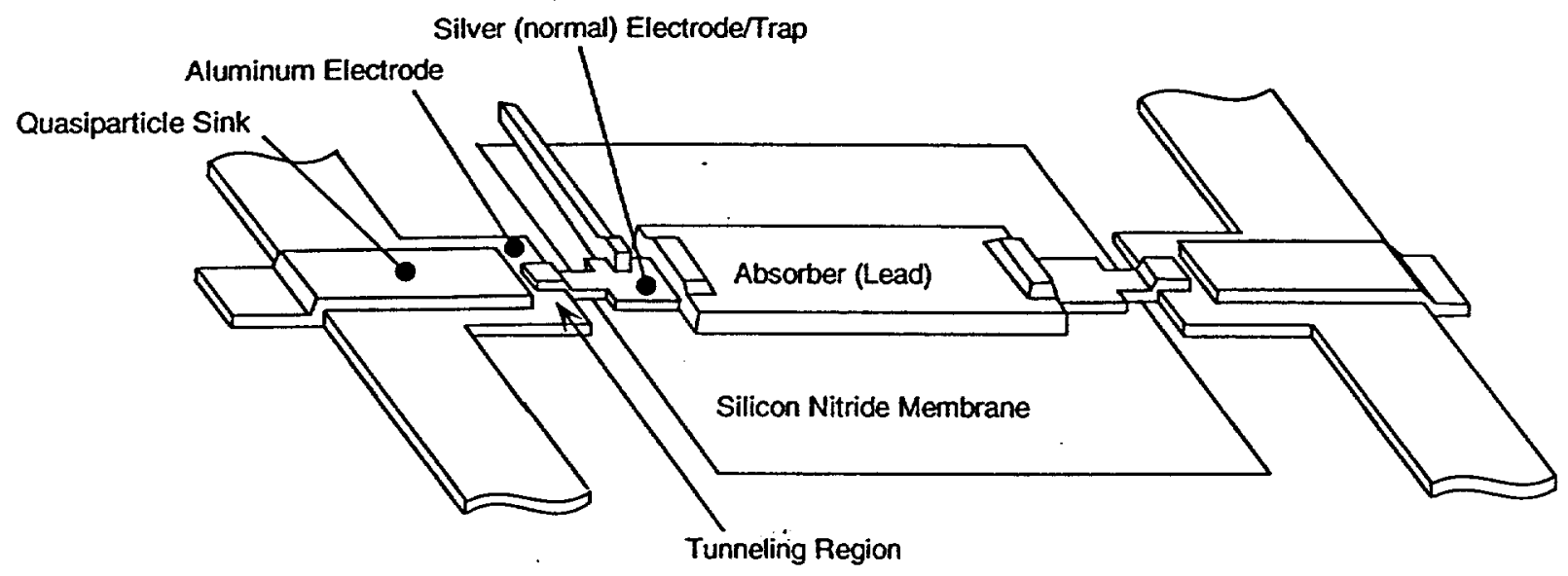

Figure 7.1. Diagram of the imaging spectrometer with HET sensors. Incoming $x$-ray photons are absorbed in the lead absorber, where they create quasiparticles. The quasiparticles diffuse through the lead until they reach the overlap region between the absorber and the normal metal electrodes of the HET sensors. Here, their energy is thermalized into the electron system in the normal metal and thus trapped and measured by the HET sensor. High energy phonons are prevented from escaping from the absorber by depositing it on a thin silicon nitride membrane. Any phonons that leave the absorber are immediately reflected back into it, giving them another chance to break Cooper pairs into quasiparticles. 


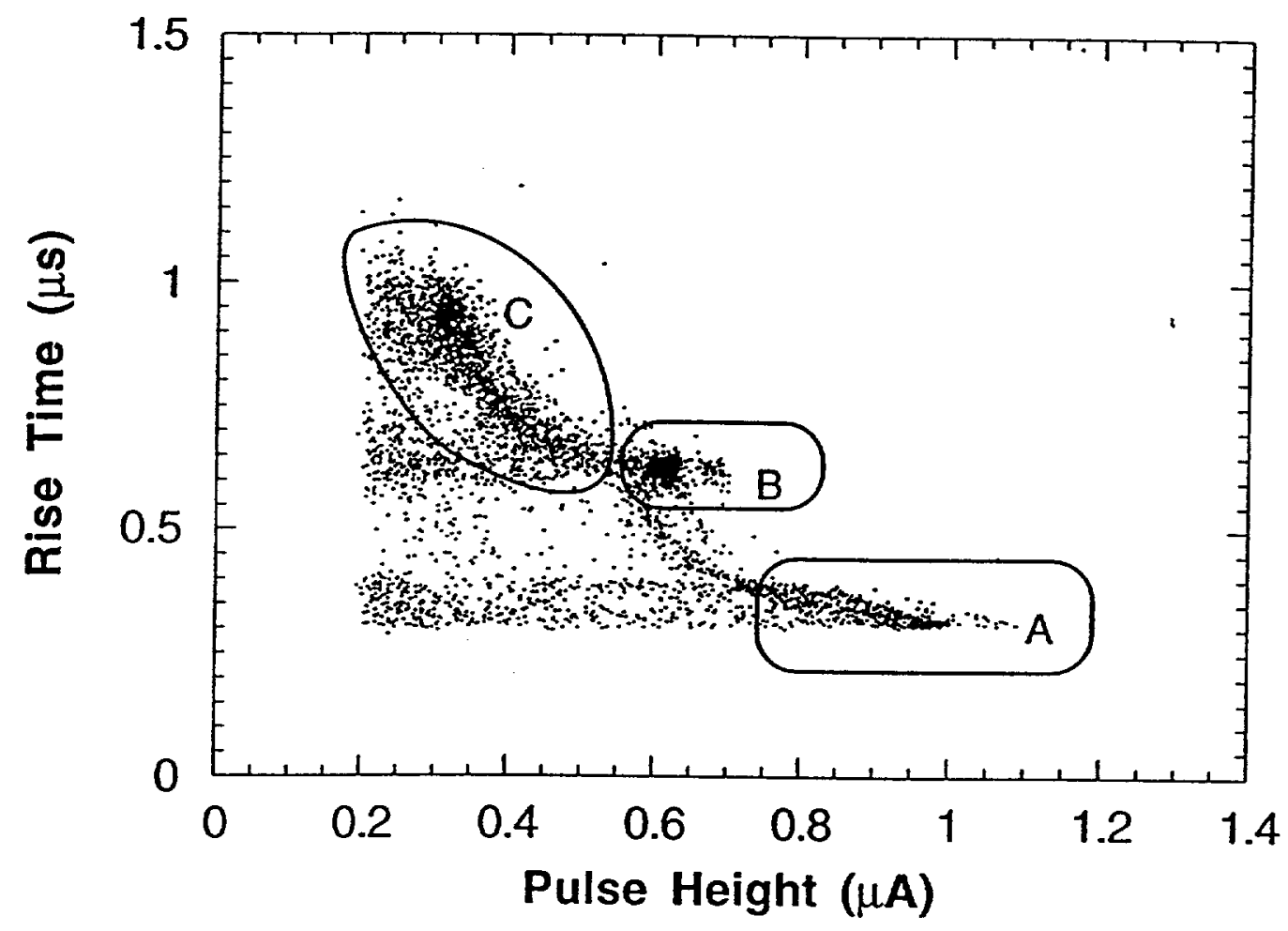

Figure 7.2. Scatter plot of rise time as a function of pulse height for 5000 typical pulses from one sensor of the imaging spectrometer. These pulse were measured at a bias voltage of $92 \mathrm{mV}$, and a temperature of $80 \mathrm{mK}$. See text for discussion of the various regions denoted in the figure. 
By considering the number of measured pulses in each region, and the thickness and area, and therefore the expected number of absorption events in different area, we are able to identify pulses due to different regions.

In Figure 7.2, pulses from region $\mathrm{A}$ are due to $\mathrm{X}$-ray photons that are absorbed directly in the region underneath the tunnel barrier in the sensor. The pulses are the largest because little or no energy is lost before the tunneling process begins. Pulses from region B are due to X-rays that are absorbed in the overlap region between the lead absorber and the normal metal electrode. These pulses have longer rise times because of the time it takes the hot electrons to diffuse from the absorption site to the tunneling region. The pulse height is less because the arrival of the energy in the tunneling region is spread out over more time, and because some energy may be lost to phonons during the diffusion process. The even smaller, longer pulses in region $C$ are due absorption events in the lead absorber. As we move further from the overlap region into the absorber, the pulses reduce in height and have even longer rise times. This produces the sloping distribution in region C. Pulses to the left of these regions are due to absorption events in the substrate underneath the various regions of the detector. In this case, the metal films in the detector absorb athermal phonons produced by the absorption events. These phonons create electronic excitations in the films similar to those produced by direct absorption, but because less energy is absorbed by the films in this case, the pulse finally produced has a reduced amplitude.

By analyzing the distribution of pulses in region $C$, we are able to deduce that for the lead films in these samples, the $\mathrm{X}$-ray induced quasiparticles only produce a measurable signal for absorption events within $\sim 150 \mu \mathrm{m}$ of the normal metal electrode. This is confirmed by the absence of simultaneous pulses from both sensors in the detector. This indicates that the quality of the absorbing material must be significantly improved in order to make a useful detector. One approach is to make the absorber out of a sample of an ultra pure superconducting crystal of a suitable material such as niobium or tantalum. We discuss the use of HET sensors in this configuration in the next section.

\section{Gamma-Ray detectors}

\section{A. Introduction}

One of the goals of our research is to produce high energy resolution spectrometers for gamma rays. For gamma rays, the quantum efficiency of a high resolution detector based on superconducting thin films would be far too low to be a useful detector. Instead, we plan to use bulk samples of high purity superconducting crystals as absorbing elements. These will be coupled to cryogenic quasiparticle sensors to collect and measure the excitations produced by the absorption of the gamma-ray photon. Because of the large volume of these gamma-ray absorbers, quasiparticles must first be trapped and concentrated into an intermediate level trap, and then concentrated further into a material with an even lower energy gap. The normal electrode HET sensor is ideal for this final 
trapping region, since it has zero energy gap, and therefore can efficiently trap quasiparticles from the intermediate level trap. In this section, we discuss our efforts to develop a fabrication process for HET sensors compatible with their production on top of superconducting crystals.

\section{B. Device Design and Fabrication}

A schematic cross-section of one of our $\mathrm{Ag} / \mathrm{Al}_{2} \mathrm{O}_{3} / \mathrm{Al}$ NIS tunnel junctions is shown in Fig. 8.1. All the metal films are deposited by DC magnetron sputtering and structured using $\mathrm{BeCu}$ shadow masks. These masks are similar to those used in sections, but are used in our ultra-high vacuum film deposition system dedicated to the production of gamma-ray detectors. In these first test structures, a $200 \mathrm{~nm}$-thick film of aluminum takes the place of the superconducting crystal. However, all fabrication steps are compatible with fabrication of the sensor on a crystalline sample.

The first fabrication step is the deposition of a $200 \mathrm{~nm}$ thick Al base film. A $200 \mathrm{~nm}$ thick $\mathrm{Ag}$ base electrode is then deposited, followed by a $20 \mathrm{~nm}$ thick Al seed layer. The sample is oxidized in the load-lock for 30 minutes at an oxygen pressure of $1.0 \mathrm{Tor}$, after which the $200 \mathrm{~nm}$ thick Al counter electrode is deposited. The next step is the deposition of an insulating layer which electrically isolates the wiring layer from the base film and has small holes to allow contact to the counter electrodes of the junctions. A $700 \mathrm{~nm}$ thick thermally evaporated layer of $\mathrm{SiO}$ is deposited, and the holes are defined by means of liftoff. The last step is the deposition of the $500 \mathrm{~nm}$ thick Al wiring layer and the $200 \mathrm{~nm}$ thick $\mathrm{Al}$ film that makes contact to the Al base film.

\section{Preliminary Results}

To test this fabrication process we fabricated both $\mathrm{Ag} / \mathrm{Al}_{2} \mathrm{O}_{3} / \mathrm{Al}$ (NIS) tunnel junctions and $\mathrm{Al} / \mathrm{Al}_{2} \mathrm{O}_{3} / \mathrm{Al}$ (SIS) tunnel junctions. The $\mathrm{Al} / \mathrm{Al}_{2} \mathrm{O}_{3} / \mathrm{Al}$ devices were basically the same as the one shown in Fig. 8.1. The only difference is that the $\mathrm{Ag}$ base electrode and the Al seed layer are left out of the device and thus the device was fabricated directly on the bottom aluminum layer. We use the ratio $\mathrm{RD}$, the dynamic resistance in the subgap region measured at $\sim 60 \mathrm{mK}$, to $\mathrm{RN}$, the normal state resistance of the tunnel junction, to parameterize the junction quality. The $\mathrm{Al} / \mathrm{Al}_{2} \mathrm{O}_{3} / \mathrm{Al}$ devices showed typical SIS I-V characteristics with quality factors $R_{D} / R_{N}$ up to $4.6 \cdot 10^{5}$.

The $\mathrm{Ag} / \mathrm{Al}_{2} \mathrm{O}_{3} / \mathrm{Al}$ tunnel junctions fabricated with this new process were also very good. We measured quality factors ranging from $10^{3}$ up to $5.4 \cdot 10^{4}$. A typical I-V characteristic for a $100 \times 100 \mu \mathrm{m}^{2} \mathrm{Ag} / \mathrm{Al}_{2} \mathrm{O}_{3} / \mathrm{Al}$ device is shown in Fig. 8.2 (a). This curve was measured at $T=65 \mathrm{mK}$ and in zero applied magnetic field. The measured $R_{N}$ is $0.19 \Omega$. The I-V characteristic clearly shows the appearance of a small supercurrent of approximately $350 \mu \mathrm{A}$. Thus instead of a pure NIS tunnel junction we have an S'IS junction. Due to the proximity effect the $\mathrm{Ag}$ base electrode no longer behaves as a normal metal. The Al base film underneath the Ag induces an energy gap $\Delta_{\mathrm{Ag}}$ of $50 \mu \mathrm{eV}$. 


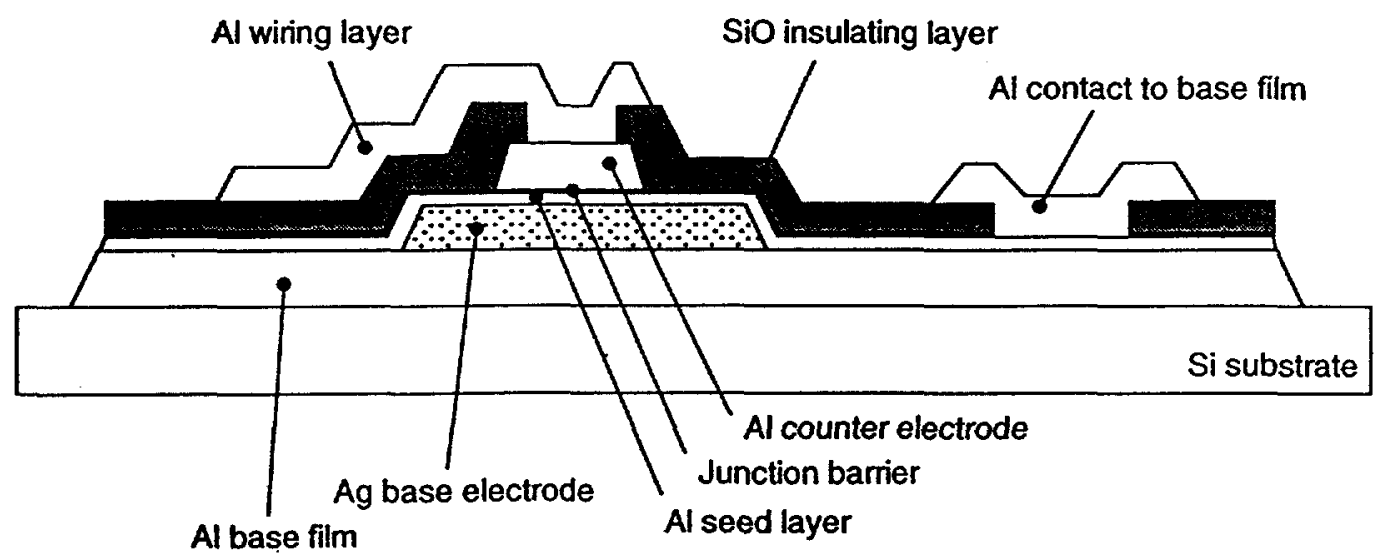

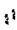

Figure 8.1 Cross stection of an HET sensor made with a process that is compatible with fabrication directly on top of a crystallinesuperconducting gamma-ray absorber. In this preliminary device, a "fake" single crystal is used that is made from an aluminum thin film. In a real gamma-ray spectrometer, this film would be replacedby a single-crystal tantalum gammaray absorber. Gamma-ray induced excitations will be trapped in the normal metal electrodes of the HET sensor, where they are measured. Fabrication details are discussed in the text. 
This effect can clearly be seen in Fig. 8.2 (b) where only the subgap region is shown in zero applied magnetic field at temperatures of $75,200,250,300$ and $425 \mathrm{mK}$. In these I$\mathrm{V}$ characteristics one can clearly see the current steps at the bias voltages of $50 \mu \mathrm{V}$ corresponding to $\mathrm{V}_{\text {bias }}=\Delta_{\mathrm{Ag}} / \mathrm{e}, 130 \mu \mathrm{V}$ corresponding to $\mathrm{V}_{\text {bias }}=\left(\Delta_{\mathrm{Ag}}-\Delta_{\mathrm{A}}\right) / \mathrm{e}$ and $230 \mu \mathrm{V}$ corresponding to $V_{\text {bix }}=\left(\Delta_{\mathrm{Ag}}+\Delta_{\mathrm{A}}\right) / \mathrm{e}$. When the temperature is increased it can be seen that $\Delta_{\mathrm{A}}$ is reduced until at $\mathrm{T}=430 \mathrm{mK}$ the $\mathrm{Ag}$ base electrode becomes normal. In Fig. 8.2 (c) we show I-V characteristics measured at $\mathrm{T}=180 \mathrm{mK}$ and at different applied magnetic fields of $B=2,3,3.5,4$ and $4.5 \mathrm{mT}$. We can clearly see that the $\mathrm{Ag}$ base electrode is driven normal by the applied magnetic field and that at $\mathrm{B}=4.5 \mathrm{mT}$ we have a pure NIS tunnel junction.

\section{Fabrication on Crystalline samples}

Encouraged by these results, we attempted to fabricated junctions directly on top of polished samples of single-crystal tantalum. These samples we cut from a larger crystal, and then mechanically polished to a surface roughness of better that $10 \mathrm{~nm}$ r.m.s. Junctions were fabricated on this polished surface using a process identical to the one discussed above. Unlike the junctions fabricated on thin films, these junctions exhibited very high leakage resistance. We suspect that this poor junction quality is related to surface damage caused by the mechanical polishing step. Other workers have been able to fabricate high quality SIS junctions on tantalum crystals by following the mechanical polishing steps with a chemical polish. We are currently investigating this approach.

\section{Conclusions}

In this work we have studied the use of hot-electron tunneling sensors as sensing elements in cryogenic X-ray and gamma-ray spectrometer. We have nalyzed the operation of these devices, and have explained their behavior in detail. High resolution operation of the sensors is complicated by the presence of a previously undocumented heating mechanism in the normal-insulating-superconducting tunnel junction that makes up the sensor. We have proposed and studied several methods of eliminating this problem. We have also begun to integrate the HET sensor into X-ray and gamma-ray spectrometers with absorbing elements large enough to be useful in the scientific and technical community. Although our LDRD funding has ended, we are sufficiently encouraged about the prospects for these sensors to continue developing them in the context of gamma-ray spectrometers for projects funded directly by the DOE. 


\section{References}

1. H. R. Ott and A. Zehnder, Low Temperature Detectors, North-Holland (1996), Amsterdam.

2. C. A. Mears, Simon E. Labov and A. T. Barfknecht, Applied Physics Letters, 63, (1993) 2961-2963.

3. C. A. Mears, et al., Nuclear Instruments and Methods in Physics Research, A 370 , (1996) 53-56.

4. R. L. Kelley, et al., Journal of Low Temperature Physics, 93, (1993) 225-230.

5. D. McCammon, et al., Nuclear Instruments and Methods in Physics Research, A326, (1993) 157-165.

6. H. Kraus, et al., Physical Review B, 231, (1989) 195-202.

7. M. Nahum, P. L. Richards and C. A. Mears, IEEE Transactions on Applied Superconductivity, 3, (1993) 2124-2127.

8. M. Nahum, John M. Martinis and S. Castles, Journal of Low Temperature Physics, 93, (1993) 733-738.

9. N. E. Booth, Applied Physics Letters, 50, (1987) 293-295.

10. Albert Schmid, Z Physik, 259, (1973) 421-436.

11. Albert Schmid, Z Physik, 271, (1974) 251-256.

12. Richard P. Welty and John M. Martinis, IEEE Transactions on Applied

Superconductivity, 3, (1993) 2605-2608.

13. M Nahum, T. M. Eiles and John Martinis, Applied Physics Letters, 65, (1994) 3123-3125.

14. Michael Tinkham, Physical Review B, 6, (1972) 1747-1756. 


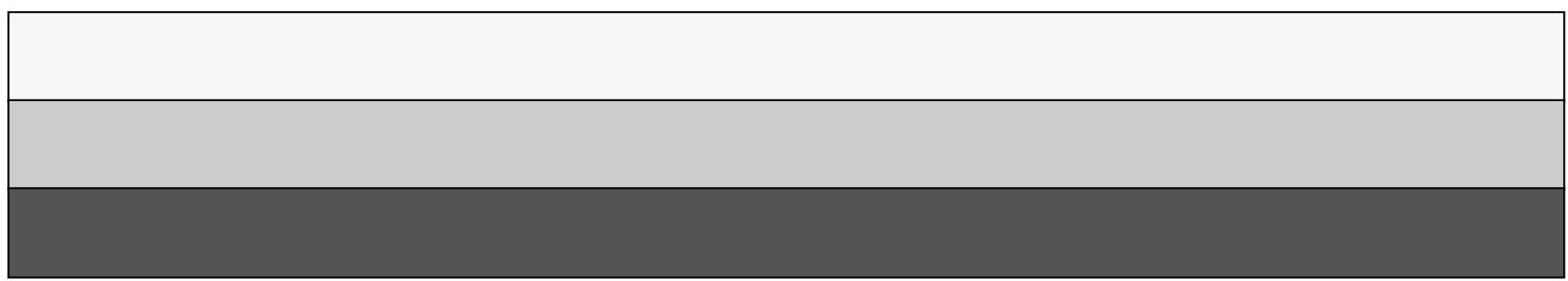

